\title{
On the nonreflecting boundary operators for the general two dimensional Schrödinger equation
}

Vishal Vaibhav ${ }^{\mathrm{a}}$

Of the two main objectives we pursue in this paper, the first one consists in the studying operators of the form $\left(\partial_{t}-i \Delta_{\Gamma}\right)^{\alpha}, \alpha=1 / 2,-1 / 2,-1, \ldots$, where $\Delta_{\Gamma}$ is the Laplace-Beltrami operator. These operators arise in the context of nonreflecting boundary conditions in the pseudo-differential approach for the general Schrödinger equation. The definition of such operators is discussed in various settings and a formulation in terms of fractional operators is provided. The second objective consists in deriving corner conditions for a rectangular domain in order to make such domains amenable to the pseudo-differential approach. Stability and uniqueness of the solution is investigated for each of these novel boundary conditions.

\section{INTRODUCTION}

In this article, we consider the problem of construction of nonreflecting boundary condition for the general two dimensional Schrödinger equation. In particular, we consider the following initial value problem (IVP):

$$
\begin{aligned}
& i \partial_{t} u+\Delta u+\phi\left(\mathbf{x}, t,|u|^{2}\right) u=0, \quad(\mathbf{x}, t) \in \mathbb{R}^{2} \times \mathbb{R}_{+}, \\
& u(\mathbf{x}, 0)=u_{0}(\mathbf{x}), \quad \mathbf{x} \in \mathbb{R}^{2} .
\end{aligned}
$$

where $\mathbb{R}_{+}$denotes the non-negative real numbers $(\mathbb{R})$ and the initial data is assumed to be supported within the computational domain, $\Omega_{i}$, i.e., supp $u_{0} \subset \Omega_{i}$. Let the boundary of the computational domain be denoted by $\Gamma$. Further, we assume that the potential function, $\phi$, is real-valued. This problem has been treated by several authors ${ }^{1-10}$. Exact formulations of the transparent boundary condition (TBC) for the free Schrödinger equation on convex domains, $\Omega_{i}$, with smooth boundary was provided by Schädle ${ }^{4}$ in terms of a single and a double layer potential. The special case of a circular domain was treated by Han and Huang ${ }^{5}$. Earlier attempts to derive an exact TBC for a rectangular domain by Menza proved to be problematic on account of the presence of corners ${ }^{1,2}$. This problem is resolved in a recent work by Feshchenko and Popov ${ }^{8}$. For the spatially discretized free Schrödinger equation, TBCs on a rectangular domain have also been recently reported by Ji et al. ${ }^{11}$ where exact form of the Green's function was obtained on a purely discrete level to construct the discrete TBCs. The stability analysis of these discrete TBCs is carried out in Ref. 12. On account of the lack of integrability, these techniques cannot be applied to the general Schrödinger equation and one has to turn to approximate methods (see Refs. 13 and 14 for a comprehensive literature survey).

For the approximate methods, we restrict ourselves to the pseudo-differential approach (in particular, the gauge transformation strategy ${ }^{14}$ ) for constructing approximate nonreflecting boundary conditions referred to as the absorbing boundary conditions or artificial boundary conditions for various types of computational domains. Our goal is to understand operators of the form $\left(\partial_{t}-i \triangle_{\Gamma}\right)^{\alpha}, \alpha=1 / 2,-1 / 2,-1, \ldots$, which appeared in the works of Menza ${ }^{1,2}$ and Antoine et al $^{6,9}$. Several aspects of such artificial boundary conditions (ABCs) which comprises these operators are not quite well understood; we discuss these issues which motivate the present work in the subsequent paragraphs.

Contrary to the existing belief that the aforementioned operators can only be implemented via a Padé approximation ${ }^{15}$ of a monomial of fractional degree $\alpha$, i.e., $z^{\alpha}$, it is shown in various settings that this operator can be expressed in terms of fractional operators. For arbitrary functions

\footnotetext{
a)vishal.vaibhav@gmail.com
} 
$f(x, t),(x, t) \in \mathbb{R} \times \mathbb{R}_{+}$, the operation $\left(\partial_{t}-i \triangle_{x}\right)^{\alpha} f(x, t), \alpha=1 / 2,-1 / 2,-1, \ldots$, requires the knowledge of the function over its entire support. If $\operatorname{supp}_{x} f(x, t) \subset \Gamma_{x}, \forall t \geq 0$ or $f(x, t)$ is periodic with respect to $x$ and it can be uniquely defined by its values at $x \in \Gamma_{x}$ for all $t \geq 0$, then it is shown that a formulation particularly convenient for expressing the TBCs/ABCs for the IVP in (1) can be developed ${ }^{16}$. Let us remark that the numerical implementation of such operators is not being presented in this paper; however, it can be easily seen that the formulation developed in this paper makes these operators amenable to convolution quadrature ${ }^{17}$. It might be expected that the new scheme affords improvement in accuracy over the existing Padé approximation based method ${ }^{18}$ reported in Refs. 10 and 19.

Further, it is well known that the pseudo-differential approach cannot be applied to computational domains with corners. This precludes the rectangular domain which happens to be a very convenient choice of the computational domain. The ABCs involving the operators of the form $\left(\partial_{t}-i \triangle_{\Gamma}\right)^{\alpha}$ cannot be adapted to the rectangular domain in a straightforward manner; however, the ABCs obtained as local approximations (with respect to $x$ ) or, equivalently, the high-frequency approximations of this operator admit of the possibility of constructing the so-called corner conditions. We demonstrate this possibility for the free as well as the general Schrödinger equation given by (1). Our approach is closely related to the ideas presented in Refs. 20 and 21.

Another program that we have followed in this paper is of obtaining the well-known "energy" estimate which can be introduced as follows: In the context of electromagnetic fields, the square of the $\mathrm{L}^{2}$-norm is related to the total energy, $E(t)$, of the field given by

$$
E(t ; \Omega)=\int_{\Omega}|u(\mathbf{x}, t)|^{2} d^{2} \mathbf{x}=\|u(\cdot, t)\|_{L^{2}(\Omega)}^{2}, \quad \forall t \geq 0 .
$$

The total energy, $E\left(t ; \mathbb{R}^{2}\right)$, remains constant in a conservative system which is also true of (1) given that $\phi$ is real-valued. If the initial field is supported in $\Omega_{i}$, the fact that the energy content of the field in $\Omega_{i}$, at any later time $t>0$, cannot exceed that of the initial field is expressed by the inequality $E\left(t ; \Omega_{i}\right) \leq E\left(0 ; \Omega_{i}\right)$ or, equivalently,

$$
\|u(\cdot, t)\|_{\mathrm{L}^{2}\left(\Omega_{i}\right)} \leq\left\|u_{0}\right\|_{\mathrm{L}^{2}\left(\Omega_{i}\right)}, \quad \forall t>0,
$$

for the IVP in (1) with TBCs/ABCs as boundary conditions involving operators of the form $\left(\partial_{t}-\right.$ $\left.i \triangle_{\Gamma}\right)^{\alpha}$ or high-frequency approximations of $\mathrm{it}^{22}$. Under the assumption that the solution exists, this inequality guarantees the stability as well as the uniqueness of the solution of the equivalent initial boundary-value problem (IBVP). In certain cases, this result can be obtained by resorting to a general construct such that for any pseudo-differential operator, $P$, and a function $u \in \mathrm{C}_{0}^{\infty}(\Omega)$ we have

$$
2 \operatorname{Re}\langle u \mid P u\rangle=\langle u \mid P u\rangle+\left\langle u \mid P^{\dagger} u\right\rangle
$$

where $P^{\dagger}$ is adjoint of the operator $P, \operatorname{Re}$ stands for real part and $\langle u \mid v\rangle=\int_{\Omega} u^{*} v d \Omega$. Given the symbol $\sigma_{P}$ of $P$, the symbol of the adjoint, $\sigma_{P^{\dagger}}$, can be computed using the following general formula: Let $\mathbb{N}^{n}$ denote the $n$-ary Cartesian power of set of non-negative integers. Assuming $y \in \mathbb{R}^{n}$ and $\zeta$ the covariable of $y$, we have

$$
\sigma_{P^{\dagger}}(y, \zeta) \sim \sum_{\alpha \in \mathbb{N}^{n}} \frac{1}{\alpha ! i^{|\alpha|}} \partial_{\zeta}^{\alpha}\left[\partial_{y}^{\alpha} \sigma_{P}^{*}(y, \zeta)\right]
$$

where $\sigma_{P}^{*}$ stands for complex conjugate of $\sigma_{P}$ (complex conjugate of $z \in \mathbb{C}$ is also denoted by $\bar{z}$ ). A more detailed discussion of this approach is provided in the Appendix A. Let us note that (5) determines $\sigma_{P^{\dagger}}$ as an asymptotic series; therefore, the energy estimates obtained by retaining only the leading order terms in (5) holds only in a asymptotic sense. Given that it is usually a formidable task to demonstrate the stability of the IBVP involving TBCs/ABCs, it appears to be a more realistic goal to establish this "weak" form of stability ${ }^{23}$.

The discussion of the primary results in this paper is broadly divided into two sections: Section II deals with the free Schrödinger equation while Section III deals with the general Schrödinger equation. For each of these problems, we consider two types of domains, namely, the rectangular domain (or, infinite strip with periodic boundary condition along the unbounded direction) 
and convex domains with smooth boundary. The basic definition of the operator $\left(\partial_{t}-i \triangle_{\Gamma}\right)^{\alpha}, \alpha=$ $1 / 2,-1 / 2,-1, \ldots$, is discussed in various settings. Two families of ABCs are considered in this paper: first one obtained via the standard pseudo-differential approach and the second one obtained as the high-frequency approximation of the former. The derivation of corner conditions and the study of stability and uniqueness of the solution are carried out separately for each of these problems in the subsections.

\section{FREE SCHRÖDINGER EQUATION}

Let us start our discussion with the linear case of the equation (1) with null-potential, i.e.,

$$
\begin{aligned}
& i \partial_{t} u+\Delta u=0, \quad(\mathbf{x}, t) \in \mathbb{R}^{2} \times \mathbb{R}_{+}, \\
& u(\mathbf{x}, 0)=u_{0}(\mathbf{x}), \quad \mathbf{x} \in \mathbb{R}^{2} .
\end{aligned}
$$

The problem of constructing transparent boundary condition can be exactly treated for the case of compactly supported initial data for a computational domain $\Omega_{i}$ with a general smooth boundary ${ }^{4}$, say, $\Gamma$. The basic approach involves solving the initial boundary value problem on the exterior domain which is defined by $\Omega_{e}=\mathbb{R}^{2} \backslash \bar{\Omega}_{i}$. It is known that a boundedness condition at infinity does not ensure that a unique solution of the IVP in (6) exists; one additionally needs to impose a Sommerfeld-like radiation condition at infinity in order to exclude all the incoming waves from infinity. This condition reads as

$$
\lim _{|\mathbf{x}| \rightarrow \infty} \sqrt{|\mathbf{x}|}\left(\nabla u \cdot \frac{\mathbf{x}}{|\mathbf{x}|}+e^{-i \frac{\pi}{4}} \partial_{t}^{1 / 2} u\right)=0 .
$$

Using the decomposition of $u(\mathbf{x}, t) \in \mathrm{L}^{2}\left(\mathbb{R}^{2}\right)=\mathrm{L}^{2}\left(\Omega_{i}\right) \oplus \mathrm{L}^{2}\left(\Omega_{e}\right)$ and introducing the fields $v(\mathbf{x}, t)$ and $w(\mathbf{x}, t)$ we have

$$
\begin{aligned}
& \left\{\begin{array}{l}
i \partial_{t} v+\Delta v=0, \quad(\mathbf{x}, t) \in \Omega_{i} \times \mathbb{R}_{+}, \\
v(\mathbf{x}, 0)=u_{0}(\mathbf{x}), \quad \mathbf{x} \in \Omega_{i} ; \\
i \partial_{t} w+\Delta w=0, \quad(\mathbf{x}, t) \in \Omega_{e} \times \mathbb{R}_{+}, \\
u(\mathbf{x}, 0)=0, \quad \mathbf{x} \in \Omega_{e}, \\
\lim _{|\mathbf{x}| \rightarrow \infty} \sqrt{|\mathbf{x}|}\left(\Delta w \cdot \frac{\mathbf{x}}{|\mathbf{x}|}+e^{-i \frac{\pi}{4}} \partial_{t}^{1 / 2} w\right)=0
\end{array}\right. \\
& \left.v(\mathbf{x}, t)\right|_{\Gamma}=\left.w(\mathbf{x}, t)\right|_{\Gamma},\left.\partial_{n} v(\mathbf{x}, t)\right|_{\Gamma}=\left.\partial_{n} w(\mathbf{x}, t)\right|_{\Gamma} .
\end{aligned}
$$

Construction of the nonreflecting boundary conditions involves solving the exterior problem exactly. In the following sections, we first consider a infinite strip as computational domain (or periodic boundary condition in the direction it extends to infinity) then extend the results to a rectangular domain.

\section{A. Infinite strip}

In this section, we restrict ourself to the case of an infinite strip with the boundary parallel to one of the coordinate axes, or, rectangular domain with a periodic boundary condition along one of the coordinate axes. Let the coordinate axes be labelled as $\mathbf{e}_{1}$ and $\mathbf{e}_{2}$. For the infinite strip, say, with a boundary parallel to the axis $\mathbf{e}_{2}$, we assume that $\operatorname{supp}_{x_{1}} u_{0}(\mathbf{x})$ is bounded. The derivation of the TBCs for the infinite straight boundary is particularly simple and can be obtained using Laplace transform in time and a Fourier transform in space. Let us consider the IVP in (6) where the domain is defined by the infinite strip between $x_{1}=x_{l}$ and $x_{1}=x_{r}$ (see Fig. 1). For the TBC on the right boundary, one must consider the IBVP on the right exterior domain $\Omega_{r}=\left(x_{r}, \infty\right) \times \mathbb{R}$. Let the covariables of $\left(x_{1}, x_{2}\right)$ 


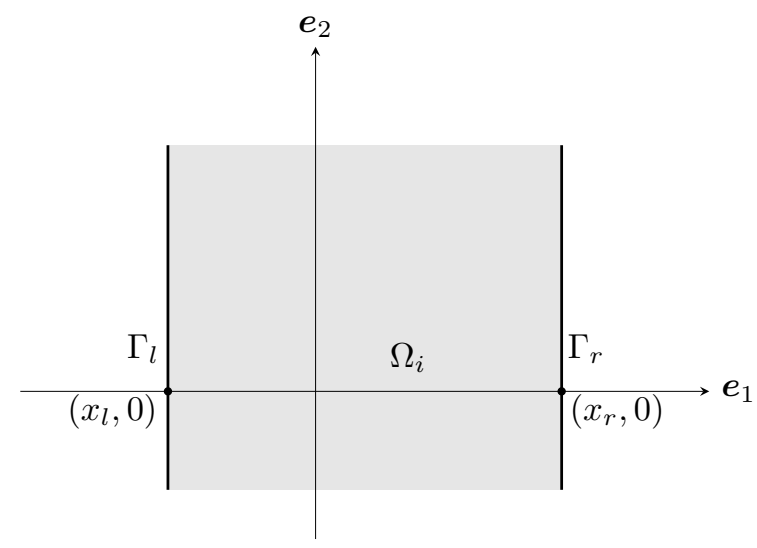

FIG. 1. The figure shows the computational domain, $\Omega_{i}$, which is an infinite strip with boundaries parallel to the axis $\mathbf{e}_{2}$. This domain can be replaced by a rectangular domain if we assume periodic boundary condition along the unbounded direction.

be denoted by $\left(\zeta_{1}, \zeta_{2}\right)$, respectively. We introduce the notation $\mathscr{F}_{x_{1}} f\left(x_{1}, x_{2}, t\right)=\mathscr{F}_{x_{1}}[f]\left(\zeta_{1}, x_{2}, t\right)$ for one dimensional Fourier transform with respect to $x_{1}$ (similarly $\mathscr{F}_{x_{2}} f\left(x_{1}, x_{2}, t\right)=\mathscr{F}_{x_{2}}[f]\left(x_{1}, \zeta_{2}, t\right)$ for Fourier transform with respect to $x_{2}$ ). For denoting the Laplace transform of a function of $t$, we use $\mathscr{L}_{t} f(t)=\mathscr{L}_{t}[f](s)=F(s)$.

Let us denote the Fourier transform with respect $x_{2}$ of $w\left(x_{1}, x_{2}, t\right)$ by $\widetilde{w}\left(x_{1}, \zeta_{2}, t\right)$ and Laplace transform with respect to $t$ of $\widetilde{w}\left(x_{1}, x_{2}, t\right)$ by $\widetilde{W}\left(x_{1}, \zeta_{2}, s\right)$, i.e.,

$$
\begin{aligned}
& \widetilde{w}\left(x_{1}, \zeta_{2}, s\right)=\mathscr{F}_{x_{2}} w\left(x_{1}, x_{2}, t\right), \\
& \widetilde{W}\left(x_{1}, \zeta_{2}, s\right)=\mathscr{L}_{t} \widetilde{w}\left(x_{1}, \zeta_{2}, t\right) .
\end{aligned}
$$

For the case of compactly supported initial data, we have

$$
\left(\partial_{x_{1}}^{2}+\alpha^{2}\right) \widetilde{W}\left(x_{1}, \zeta_{2}, s\right)=0, \quad x_{1} \in\left(x_{r}, \infty\right)
$$

where $\alpha=\sqrt{i s-\zeta_{2}^{2}}$ such that $\operatorname{Im}(\alpha)>0$. The solution can be worked out as follows: observing

$$
\begin{aligned}
& \partial_{x_{1}} \widetilde{W}\left(x_{1}, \zeta_{2}, s\right)=i \alpha \widetilde{W}\left(x_{1}, \zeta_{2}, s\right), \\
& \mathscr{L}^{-1}\left[\partial_{x_{1}} \widetilde{W}\left(x_{1}, \zeta_{2}, s\right)\right]=\mathscr{L}^{-1}\left[i \alpha^{-1}\right] \star \mathscr{L}^{-1}\left[\alpha^{2} \widetilde{W}\left(x_{1}, \zeta_{2}, s\right)\right],
\end{aligned}
$$

where ' $\star$ ' represents the convolution operation, we have

$$
\partial_{x_{1}} \widetilde{w}\left(x_{1}, \zeta_{2}, t\right)=e^{i \pi / 4} e^{-i \zeta_{2}^{2} t} \partial_{t}^{-1 / 2} e^{i \zeta_{2}^{2} t}\left[i \partial_{t} \widetilde{w}\left(x_{1}, \zeta_{2}, t\right)+\left(\widetilde{\partial_{x_{2}}^{2} w}\right)\left(x_{1}, \zeta_{2}, t\right)\right]
$$

It is also easy to verify

$$
\partial_{t}\left[e^{-i \zeta_{2}^{2} t} \partial_{t}^{-1 / 2} e^{i \zeta_{2}^{2} t} \widetilde{w}\left(x_{1}, \zeta_{2}, t\right)\right]=e^{-i \zeta_{2}^{2} t} \partial_{t}^{-1 / 2} e^{i \zeta_{2}^{2} t} \partial_{t} \widetilde{w}\left(x_{1}, \zeta_{2}, t\right)
$$

Let

$$
\mathcal{G}\left(x_{2}, t\right)=\frac{e^{-i \pi / 4}}{\sqrt{4 \pi t}} e^{i \frac{x_{2}^{2}}{4 t}}, \quad t \in \mathbb{R}_{+}
$$

so that its Laplace transform reads as $\widetilde{\mathcal{G}}\left(\zeta_{2}, t\right)=e^{-i \zeta_{2}^{2} t}$. Now, taking the inverse Fourier transform in (11), we obtain the Dirichlet-to-Neumann map as ${ }^{2}$ 


$$
\begin{aligned}
\partial_{x_{1}} w(\mathbf{x}, t) & =\frac{e^{i \pi / 4}}{\sqrt{\pi}} \int_{0}^{t} \int_{\mathbb{R}}\left[i \partial_{\tau} w\left(x_{1}, x_{2}^{\prime}, \tau\right)+\partial_{x_{2}^{\prime}}^{2} w\left(x_{1}, x_{2}^{\prime}, \tau\right)\right] \frac{\mathcal{G}\left(x_{2}-x_{2}^{\prime}, t-\tau\right)}{\sqrt{t-\tau}} d x_{2}^{\prime} d \tau \\
& =-\left(\partial_{t}-i \partial_{x_{2}}^{2}\right) \frac{e^{-i \pi / 4}}{\sqrt{\pi}} \int_{0}^{t} \int_{\mathbb{R}} w\left(x_{1}, x_{2}^{\prime}, \tau\right) \frac{\mathcal{G}\left(x_{2}-x_{2}^{\prime}, t-\tau\right)}{\sqrt{t-\tau}} d x_{2}^{\prime} d \tau
\end{aligned}
$$

This map can be expressed compactly if we employ the notation

$$
\left(\partial_{t}-i \partial_{x_{2}}^{2}\right)^{-1 / 2} f\left(x_{2}, t\right)=\frac{1}{\sqrt{\pi}} \int_{0}^{t} \int_{\mathbb{R}} f\left(x_{1}, x_{2}^{\prime}, \tau\right) \frac{\mathcal{G}\left(x_{2}-x_{2}^{\prime}, t-\tau\right)}{\sqrt{t-\tau}} d x_{2}^{\prime} d \tau,
$$

where $f \in \mathrm{C}^{\infty}\left(\mathbb{R} \times \mathbb{R}_{+}\right)$belongs to the Schwartz class with respect to $x_{2}$. On account of the singular nature of the symbol, it is not a pseudo-differential operator. However, away from the points satisfying $\xi+\zeta_{2}^{2}=0$, it can be microlocally regarded as a pseudo-differential operator with a symbol $\left(i \xi+i \zeta_{2}^{2}\right)^{-1 / 2}$, with the branch cut defined by $-\pi \leq \arg \left(i \xi+i \zeta_{2}^{2}\right)<\pi$ where $\left(\zeta_{2}, \xi\right)$ are the covariables of $\left(x_{2}, t\right)$. Further, if we take the operator $\left(\partial_{t}-i \partial_{x_{2}}^{2}\right)^{1 / 2}$ to be defined by

$$
\left(\partial_{t}-i \partial_{x_{2}}^{2}\right)^{1 / 2} f=\left(\partial_{t}-i \partial_{x_{2}}^{2}\right)\left[\left(\partial_{t}-i \partial_{x_{2}}^{2}\right)^{-1 / 2} f\right]
$$

then

$$
\partial_{x_{1}} w(\mathbf{x}, t)+e^{-i \pi / 4}\left(\partial_{t}-i \partial_{x_{2}}^{2}\right)^{1 / 2} w(\mathbf{x}, t)=0
$$

for $(\mathbf{x}, t) \in \Omega_{r} \times \mathbb{R}_{+}$.

Next, we would like to obtain a more local approximation of this boundary condition valid for small times. To this end, let us consider

$$
\mathscr{L}^{-1}\left[i \alpha^{-1}\right]=\frac{1}{2 \pi} \int\left(i s-\zeta_{2}^{2}\right)^{-1 / 2} e^{s t} d s
$$

Setting $\xi=s t$, we have

$$
\begin{aligned}
\mathscr{L}^{-1}\left[\frac{i}{\alpha}\right] & =\frac{t^{-\frac{1}{2}}}{2 \pi} \int_{a+i \mathbb{R}}\left(1-\frac{\zeta_{2}^{2} t}{i \xi}\right)^{-\frac{1}{2}} \frac{e^{\xi} d \xi}{\sqrt{i \xi}} \quad(a>0) \\
& \sim \frac{t^{-\frac{1}{2}}}{2 \pi} \int_{a+i \mathbb{R}}\left(\frac{1}{(i \xi)^{\frac{1}{2}}}+\frac{\zeta_{2}^{2} t}{2(i \xi)^{\frac{3}{2}}}+\frac{3 \zeta_{2}^{4} t^{2}}{8(i \xi)^{\frac{5}{2}}}+\ldots\right) e^{\xi} d \xi \\
& \sim \frac{e^{i \pi / 4}}{\Gamma\left(\frac{1}{2}\right)} t^{-\frac{1}{2}}+\frac{e^{-i \pi / 4}}{2 \Gamma\left(\frac{3}{2}\right)} t^{\frac{1}{2}} \zeta_{2}^{2}-\frac{3 e^{i \pi / 4}}{8 \Gamma\left(\frac{5}{2}\right)} t^{\frac{3}{2}} \zeta_{2}^{4}+\ldots
\end{aligned}
$$

Taking the inverse Fourier transform, we obtain the asymptotic expansion as

$$
\partial_{x_{1}} w+e^{-i \pi / 4} \partial_{t}^{1 / 2} w-e^{i \pi / 4} \frac{1}{2} \partial_{x_{2}}^{2} \partial_{t}^{-1 / 2} w=0 \bmod \left(\partial_{t}^{-3 / 2}\right) .
$$

For the periodic case, we may take the computational domain to be $\Omega_{i}=\left(x_{l}, x_{r}\right) \times(0,2 \pi)$ so that

$$
\begin{aligned}
& w\left(x_{1}, x_{2}, t\right)=\sum_{m \in \mathbb{Z}} \widetilde{w}_{m}\left(x_{1}, t\right) e^{i m x_{2}}, \\
& W\left(x_{1}, x_{2}, s\right)=\sum_{m \in \mathbb{Z}} \widetilde{W}_{m}\left(x_{1}, s\right) e^{i m x_{2}},
\end{aligned}
$$

with $\widetilde{W}_{m}\left(x_{1}, s\right)=\mathscr{L}_{t} \widetilde{w}_{m}\left(x_{1}, t\right)$. We also recall $x_{1} \in\left(x_{r}, \infty\right)$ whereby a limiting procedure can be used to obtain the BCs at $x_{1}=x_{r}$. For the sake of simplicity, we demonstrate the procedure for the $m$-th 
Fourier component. The complete result, then, follows by superposing all the components. Putting $\alpha_{m}=\sqrt{i s-m^{2}}$, the following results can be obtained in the same manner as before:

$$
\partial_{x_{1}} \widetilde{w}_{m}\left(x_{1}, t\right)=e^{i \pi / 4} e^{-i m^{2} t} \partial_{t}^{-1 / 2} e^{i m^{2} t}\left[i \partial_{t} \widetilde{w}_{m}\left(x_{1}, t\right)-m^{2} \widetilde{w}_{m}\left(x_{1}, t\right)\right] .
$$

Following the steps in the previous case, we have

$$
\partial_{t}\left[e^{-i m^{2} t} \partial_{t}^{-1 / 2} e^{i m^{2} t} \widetilde{w}_{m}\left(x_{1}, t\right)\right]=e^{-i m^{2} t} \partial_{t}^{-1 / 2} e^{i m^{2} t} \partial_{t} \widetilde{w}_{m}\left(x_{1}, t\right),
$$

and

$$
e^{-i m^{2} t} \partial_{t}^{-1 / 2} e^{i m^{2} t}\left[i \partial_{t} \widetilde{w}_{m}\left(x_{1}, t\right)-m^{2} \widetilde{w}_{m}\left(x_{1}, t\right)\right]=i e^{-i m^{2} t} \partial_{t}^{1 / 2} e^{i m^{2} t} \widetilde{w}_{m}\left(x_{1}, t\right)
$$

Introducing

$$
\mathcal{G}\left(x_{2}, t\right)=\sum_{m \in \mathbb{Z}} e^{i m x_{2}-i m^{2} t}
$$

which is defined only in a distributional sense and using the relations above, we obtain an expression similar to (14) as follows:

$$
\begin{aligned}
\partial_{x_{1}} w(\mathbf{x}, t) & =\frac{e^{i \pi / 4}}{\sqrt{\pi}} \int_{0}^{t} \int_{0}^{2 \pi}\left[i \partial_{\tau} w\left(x_{1}, x_{2}^{\prime}, \tau\right)+\partial_{x_{2}^{\prime}}^{2} w\left(x_{1}, x_{2}^{\prime}, \tau\right)\right] \frac{\mathcal{G}\left(x_{2}-x_{2}^{\prime}, t-\tau\right)}{\sqrt{t-\tau}} d x_{2}^{\prime} d \tau \\
& =-\left(\partial_{t}-i \partial_{x_{2}}^{2}\right) \frac{e^{-i \pi / 4}}{\sqrt{\pi}} \int_{0}^{t} \int_{0}^{2 \pi} w\left(x_{1}, x_{2}^{\prime}, \tau\right) \frac{\mathcal{G}\left(x_{2}-x_{2}^{\prime}, t-\tau\right)}{\sqrt{t-\tau}} d x_{2}^{\prime} d \tau .
\end{aligned}
$$

For periodic functions, the operator defined in (15) becomes

$$
\left(\partial_{t}-i \partial_{x_{2}}^{2}\right)^{-1 / 2} f\left(x_{2}, t\right)=\frac{1}{\sqrt{\pi}} \int_{0}^{t} \int_{0}^{2 \pi} f\left(x_{1}, x_{2}^{\prime}, \tau\right) \frac{\mathcal{G}\left(x_{2}-x_{2}^{\prime}, t-\tau\right)}{\sqrt{t-\tau}} d x_{2}^{\prime} d \tau,
$$

where the kernel is defined by (23). We now turn our attention to obtaining a form of the operator $\left(\partial_{t}-i \partial_{x_{2}}^{2}\right)^{1 / 2}$ which can be numerically implemented. Using the relation in (22), we may write

$$
\begin{aligned}
\partial_{x_{1}} w(\mathbf{x}, t) & =-e^{-i \pi / 4} \sum_{m \in \mathbb{Z}} e^{-i m^{2} t} \partial_{t}^{1 / 2}\left[e^{i m^{2} t} \widetilde{w}_{m}\left(x_{1}, t\right) e^{i m x_{2}}\right], \\
& =-e^{-i \pi / 4} \partial_{t^{\prime}}^{1 / 2} \sum_{m \in \mathbb{Z}}\left[e^{-i m^{2}\left(t-t^{\prime}\right)} \widetilde{w}_{m}\left(x_{1}, t^{\prime}\right) e^{i m x_{2}}\right]_{t^{\prime}=t} .
\end{aligned}
$$

Introducing the auxiliary function $\varphi\left(x_{1}, x_{2}, t, t^{\prime}\right)$ such that

$$
\begin{aligned}
& \varphi\left(x_{1}, x_{2}, t, t^{\prime}\right)=\sum_{m \in \mathbb{Z}}\left[e^{-i m^{2}\left(t-t^{\prime}\right)} \widetilde{w}_{m}\left(x_{1}, t^{\prime}\right) e^{i m x_{2}}\right], \\
& \partial_{x_{1}} w(\mathbf{x}, t)=-\left.e^{-i \pi / 4} \partial_{t^{\prime}}^{1 / 2} \varphi\left(x_{1}, x_{2}, t, t^{\prime}\right)\right|_{t^{\prime}=t} .
\end{aligned}
$$

In order to determine all the values of the function needed to compute the non-local fractional derivative, consider the IVP given by

$$
\left\{\begin{array}{l}
{\left[i \partial_{\tau}+\partial_{x_{2}}^{2}\right] \varphi\left(x_{1}, x_{2}, \tau, \tau^{\prime}\right)=0, \quad\left(\tau, x_{2}\right) \in\left(\tau^{\prime}, t\right] \times(0,2 \pi)} \\
\varphi\left(x_{1}, 0, \tau^{\prime}, \tau^{\prime}\right)=\varphi\left(x_{1}, 2 \pi, \tau^{\prime}, \tau^{\prime}\right), \quad \tau \in\left(\tau^{\prime}, t\right] \\
\varphi\left(x_{1}, x_{2}, \tau^{\prime}, \tau^{\prime}\right)=w\left(x_{1}, x_{2}, \tau^{\prime}\right), \quad x_{2} \in(0,2 \pi)
\end{array}\right.
$$

The solution of this IVP must be obtained for all $\tau^{\prime} \in[0, t]$. This process is schematically depicted in Fig. 2 where we note that the history of the field is needed along the vertical line up to the diagonal in the $\left(\tau, \tau^{\prime}\right)$-plane. 


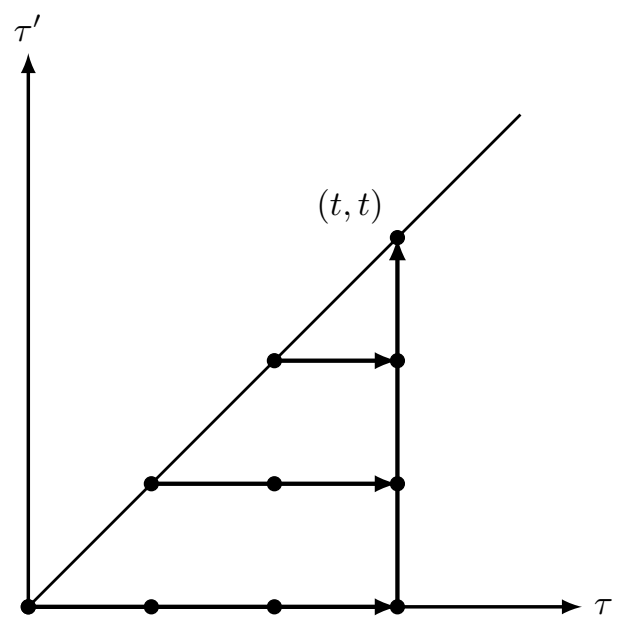

FIG. 2. In this figure, a schematic is shown illustrating how the auxiliary equation (27) will be solved in order to provide the history of the field needed in the TBCs. The field is known along the diagonal which serves as the initial conditions to obtain the values of the field needed in the TBCs (arrow in the line depicts the evolution direction in time).

Remark II.1. Such a procedure can be used to compute the action of any operator of the form $\left(\partial_{t}-i \partial_{x}^{2}\right)^{-n / 2}, n=1,2, \ldots$, on any function periodic in $x \in \mathbb{R}$, say, $f(x, t)$. Introducing an auxiliary function $\varphi\left(x, t, t^{\prime}\right)$ such that

$$
\begin{aligned}
& \varphi\left(x, t, t^{\prime}\right)=\sum_{m \in \mathbb{Z}}\left[e^{-i m^{2}\left(t-t^{\prime}\right)} \widetilde{f}_{m}\left(t^{\prime}\right) e^{i m x}\right]_{t^{\prime}=t}, \\
& \left(\partial_{t}-i \partial_{x}^{2}\right)^{-n / 2} f(x, t)=\left.\partial_{t^{\prime}}^{-n / 2} \varphi\left(x, t, t^{\prime}\right)\right|_{t^{\prime}=t} .
\end{aligned}
$$

The associated IVP is given by

$$
\left\{\begin{array}{l}
{\left[i \partial_{\tau}+\partial_{x}^{2}\right] \varphi\left(x, \tau, \tau^{\prime}\right)=0, \quad(\tau, x) \in\left(\tau^{\prime}, t\right] \times(0,2 \pi),} \\
\varphi\left(0, \tau, \tau^{\prime}\right)=\varphi\left(2 \pi, \tau, \tau^{\prime}\right), \quad \tau \in\left(\tau^{\prime}, t\right] \\
\varphi\left(x, \tau^{\prime}, \tau^{\prime}\right)=f\left(x, \tau^{\prime}\right), \quad x \in(0,2 \pi),
\end{array}\right.
$$

which needs to be solved for all $\tau^{\prime} \in[0, t]$.

\section{Stability and uniqueness}

An equivalent formulation of the IVP (6) on the computational domain $\Omega_{i}=\left(x_{l}, x_{r}\right) \times(0,2 \pi)$ with periodic boundary condition along the axis $\mathbf{e}_{2}$ is given by

$$
\left\{\begin{array}{l}
i \partial_{t} u+\Delta u=0, \quad(\mathbf{x}, t) \in \Omega_{i} \times \mathbb{R}_{+}, \\
u(\mathbf{x}, 0)=u_{0}(\mathbf{x}) \in \mathrm{L}^{2}\left(\Omega_{i}\right), \quad \text { supp } u_{0} \subset \Omega_{i}, \\
u\left(x_{1}, x_{2}+2 \pi, t\right)=u\left(x_{1}, x_{2}, t\right), \quad t>0 \\
\partial_{n} u+e^{-i \pi / 4}\left(\partial_{t}-i \partial_{x_{2}}^{2}\right)^{1 / 2} u=0, \quad \mathbf{x} \in \Gamma_{l} \cup \Gamma_{r}, t>0
\end{array}\right.
$$

Setting $d \boldsymbol{\varsigma}=\mathbf{e}_{n}|d \mathbf{x}|$ and assuming that the solution $u(\mathbf{x}, t)$ exists, we have

$$
\int_{\Omega_{i}}\left(\partial_{t}|u|^{2}\right) d^{2} \mathbf{x}=2 \operatorname{Re} \int_{\Gamma_{l} \cup \Gamma_{r}}\left(u^{*} i \nabla u\right) \cdot d \boldsymbol{\varsigma}
$$


so that

$$
\|u(\cdot, T)\|_{L^{2}\left(\Omega_{i}\right)}^{2}-\left\|u_{0}\right\|_{L^{2}\left(\Omega_{i}\right)}^{2}=\operatorname{Re} \int_{0}^{T} d t \int_{\Gamma_{l} \cup \Gamma_{r}}\left(u^{*} i \nabla u\right) \cdot d \boldsymbol{S},
$$

where for $\mathbf{x} \in \Gamma$. Further, noting $\tilde{u}_{m}\left(x_{1}, \cdot\right) \in \mathrm{H}^{1 / 4}([0, T])$ (denotes the Sobolev space $\mathrm{W}^{1 / 4,2}([0, T])$ ), we have

$$
\begin{aligned}
\mathcal{I}_{R} & =-2 \operatorname{Re} \int_{\mathbb{R}_{+}} d t \int_{\Gamma_{r}}\left(u^{*} e^{i \pi / 4} \sqrt{\partial_{t}-i \partial_{x_{2}}^{2}} u\right) d x_{2}, \\
& =-4 \pi \operatorname{Re} \sum_{m \in \mathbb{Z}_{-}} \int_{\mathbb{R}_{+}} d t \overline{\left(\tilde{u}_{m}\left(x_{r}, t\right) e^{-i m^{2} t}\right)}\left(\partial_{t}^{1 / 2} \tilde{u}_{m}\left(x_{r}, t\right) e^{-i m^{2} t}\right), \\
& =-2 \sum_{m \in \mathbb{Z}} \int_{\mathbb{R}_{-}} d \xi|\xi|^{1 / 2}\left|\mathscr{F}_{t}\left[\tilde{u}_{m}\left(x_{r}, t\right) e^{-i m^{2} t}\right]\left(x_{r}, \xi\right)\right|^{2} \leq 0 .
\end{aligned}
$$

Similarly, it can be shown that

$$
\mathcal{I}_{L}=-2 \operatorname{Re} \int_{\mathbb{R}_{+}} d t \int_{\Gamma_{l}}\left(u^{*} e^{i \pi / 4} \sqrt{\partial_{t}-i \partial_{x_{2}}^{2}} u\right) d x_{2} \leq 0 .
$$

Therefore, we have

$$
\|u(\cdot, T)\|_{L^{2}\left(\Omega_{i}\right)} \leq\left\|u_{0}\right\|_{L^{2}\left(\Omega_{i}\right)} .
$$

This result also guarantees the uniqueness of the solution.

\section{B. Rectangular domains}

Let us consider a rectangular domain given by

$$
\Omega_{i}=\left\{\left(x_{1}, x_{2}\right): x_{b}<x_{2}<x_{t}, x_{l}<x_{1}<x_{r}\right\},
$$

and denote the boundaries as $\Gamma_{l, r}=\left\{\left(x_{1}, x_{2}\right) \in \partial \Omega_{i}: x_{1}=x_{l, r}\right\}$, respectively and $\Gamma_{b, t}=\left\{\left(x_{1}, x_{2}\right) \in\right.$ $\left.\partial \Omega_{i}: x_{1}=x_{b, t}\right\}$, respectively. Assuming supp $u_{0}$ bounded in $\Omega_{i}$, the TBCs for the infinite strip cannot be taken to be the transparent boundary operators at the straight edges because the corresponding operator requires knowledge of the entire support of the field along the tangential direction at the boundary. This clearly cannot be achieved because after a certain time the field would have spread outside the domain defined by any segment of the rectangular domain, say, $\Gamma_{r}$. This issue can be resolved in the following way: Using the same notation as in the last section and observing that

$$
\begin{aligned}
& \partial_{x_{1}} \widetilde{w}\left(x_{1}, \zeta_{2}, t\right)=e^{i \pi / 4} e^{-i \zeta_{2}^{2} t} \partial_{t}^{-1 / 2} e^{i \zeta_{2}^{2} t}\left[i \partial_{t} \widetilde{w}\left(x_{1}, \zeta_{2}, t\right)+\left(\widetilde{\left.\partial_{x_{2}}^{2} w\right)}\left(x_{1}, \zeta_{2}, t\right)\right],\right. \\
& \partial_{x_{1}} \widetilde{w}\left(x_{1}, \zeta_{2}, t\right)=-\left.e^{-i \pi / 4} \partial_{t^{\prime}}^{1 / 2} e^{-i \zeta_{2}^{2}\left(t-t^{\prime}\right)} \widetilde{w}\left(x_{1}, \zeta_{2}, t^{\prime}\right)\right|_{t^{\prime}=t},
\end{aligned}
$$

and introducing the auxiliary function

$$
\mathscr{F}_{x_{2}} \varphi\left(x_{1}, x_{2}, t, t^{\prime}\right)=e^{-i \zeta_{2}^{2}\left(t-t^{\prime}\right)} \widetilde{w}\left(x_{1}, \zeta_{2}, t^{\prime}\right)
$$

so that

$$
\partial_{x_{1}} w\left(x_{1}, x_{2}, t\right)=-\left.e^{-i \pi / 4} \partial_{t^{\prime}}^{1 / 2} \varphi\left(x_{1}, x_{2}, t, t^{\prime}\right)\right|_{t^{\prime}=t} .
$$

It is easy to verify that $\varphi$ satisfies the IVP given by

$$
\begin{aligned}
& {\left[i \partial_{\tau}+\partial_{x_{2}}^{2}\right] \varphi\left(x_{1}, x_{2}, \tau, \tau^{\prime}\right)=0, \quad \in\left(\tau^{\prime}, t\right],} \\
& \varphi\left(x_{1}, x_{2}, \tau^{\prime}, \tau^{\prime}\right)=w\left(x_{1}, x_{2}, \tau^{\prime}\right),
\end{aligned}
$$




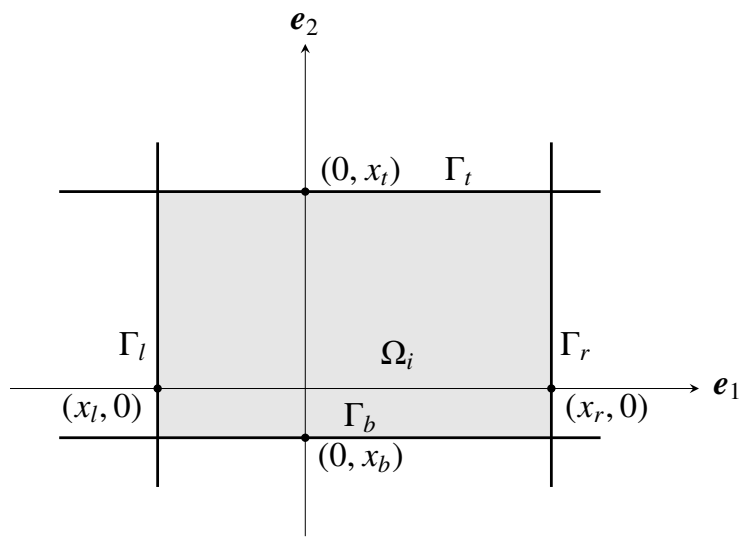

FIG. 3. The figure shows a rectangular domain with boundary segments parallel to one of the axes.
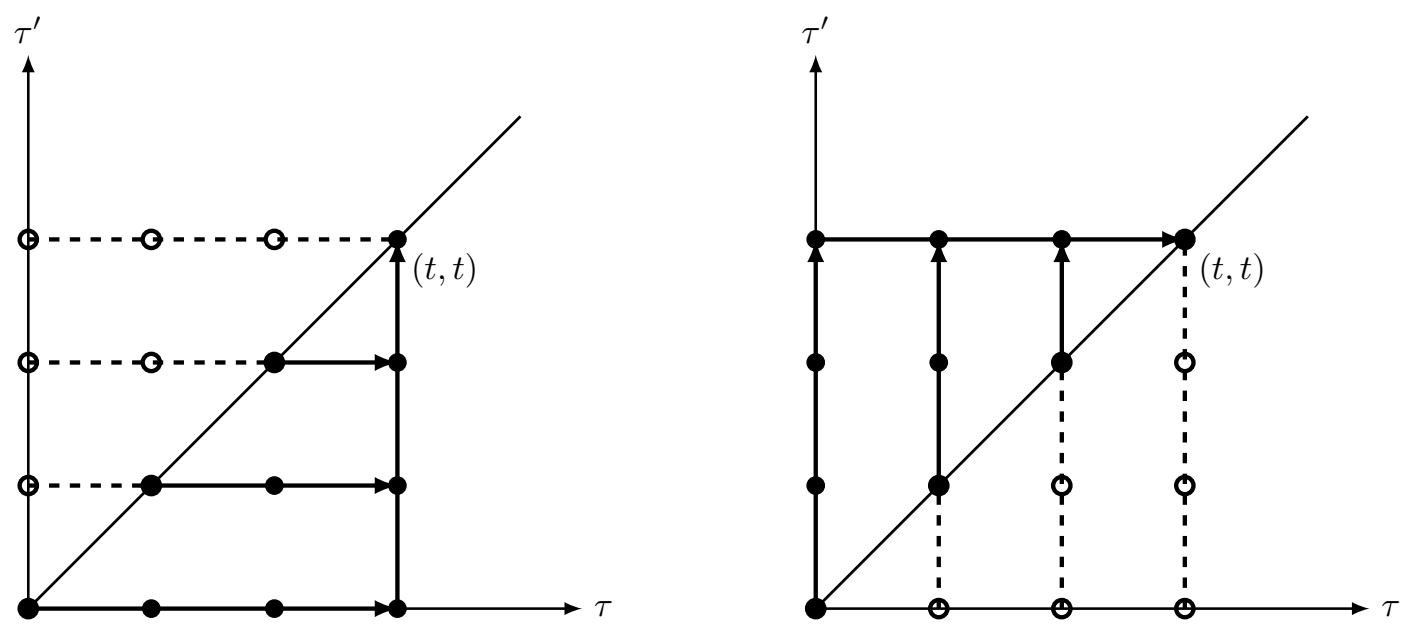

FIG. 4. A schematic depiction of the evolution of the auxiliary field $\varphi\left(x_{1}, x_{2}, \tau, \tau^{\prime}\right)$ in the $\left(\tau, \tau^{\prime}\right)$-plane is provided in this figure where the plot on the left corresponds $\mathbf{x} \in \Gamma_{r} \cup \Gamma_{l}$ and the plot on the right corresponds $\mathbf{x} \in \Gamma_{t} \cup \Gamma_{b}$. The filled circles denote the evolution of any arbitrary point that belongs to the boundary. The empty circles are relevant only when the point under consideration is a corner point. The evolution over the interior of the domain is carried either above or below the diagonal starting from the diagonal values (where direction of evolution is depicted by the arrow head). In contrast, the corner points are evolved on either side of the diagonal. Note that the vertical/horizontal lines where the arrows end corresponds to the history of the auxiliary field needed for TBCs in the current time $(t)$. The TBCs for auxiliary field require the history of the auxiliary field at the corner points, depicted by broken lines; these values are taken from the adjacent segment of the boundary.

which needs to be solved for $\tau^{\prime} \in[0, t]$ over $\Gamma_{r}$. The boundary conditions at the endpoints of the segment $\Gamma_{r}$ are not known because the original problem is defined on the infinite domain $x_{2} \in \mathbb{R}$. However, it can be shown that the restriction of the auxiliary function $\varphi\left(x_{1}, x_{2}, \tau, \tau^{\prime}\right)$ to $x_{1}=x_{r}$ at $\tau=0$ is compactly supported (with respect to $x_{2}$ ). This would allow one to impose the transparent boundary conditions at the endpoints of $\Gamma_{r}$. To this end, let us consider the IVP defined by (6), it can be solved using Fourier transform in $\left(x_{1}, x_{2}\right)$. Putting $\tilde{u}_{0}(\zeta)=\mathscr{F}_{\left(x_{1}, x_{2}\right)} u_{0}(\mathbf{x})$, we have

$$
\begin{aligned}
& \mathscr{F}_{\left(x_{1}, x_{2}\right)} u\left(\mathbf{x}, t^{\prime}\right)=e^{-i\left(\zeta_{1}^{2}+\zeta_{2}^{2}\right) \tau^{\prime}} \tilde{u}_{0}(\zeta), \\
& \varphi\left(x_{1}, x_{2}, \tau, \tau^{\prime}\right)=\frac{1}{(2 \pi)^{2}} \int_{\mathbb{R}^{2}} e^{i \zeta \cdot \mathbf{x}-i \zeta_{1}^{2} t^{\prime}-i \zeta_{2}^{2} \tau} \tilde{u}_{0}(\zeta) d^{2} \zeta
\end{aligned}
$$


Therefore, $\operatorname{supp}_{x_{2}} \varphi\left(x_{r}, x_{2}, 0, \tau^{\prime}\right) \subset\left[x_{b}, x_{t}\right]$ and

$$
\begin{aligned}
& {\left[i \partial_{\tau^{\prime}}+\partial_{x_{1}}^{2}\right] \varphi\left(x_{1}, x_{2}, \tau, \tau^{\prime}\right)=0, \quad \tau^{\prime} \in(\tau, t],} \\
& \varphi\left(x_{1}, x_{2}, \tau, \tau\right)=w\left(x_{1}, x_{2}, \tau\right), \quad \tau \in[0, t] .
\end{aligned}
$$

Hence, the boundary condition at $\left(x_{1}, x_{2}\right) \in \partial \Gamma_{r}$ is given by

$$
\partial_{x_{2}} \varphi\left(x_{1}, x_{2}, \tau, \tau^{\prime}\right) \pm e^{-i \pi / 4} \partial_{\tau}^{1 / 2} \varphi\left(x_{1}, x_{2}, \tau, \tau^{\prime}\right)=0
$$

where the sign is determined by $x_{2} \in\left\{x_{t}, x_{b}\right\}$, respectively. This requires the knowledge of $\varphi\left(x_{1}, x_{2}, \tau, \tau^{\prime}\right)$ at $\left(x_{1}, x_{2}\right) \in \partial \Gamma_{r}$ and $0 \leq \tau \leq \tau^{\prime}$. The algorithm can be explained by means of the Fig. 4. There are two IVPs for the auxiliary field $\varphi\left(x_{1}, x_{2}, \tau, \tau^{\prime}\right)$ each of which evolve the field either above or below the diagonal in the $\left(\tau, \tau^{\prime}\right)$-plane starting from the values at the diagonal in their respective domains. The filled circles denote the evolution of any arbitrary point of the boundary and the arrows denote the direction of evolution. All diagonal points are evolved along $\tau$ or $\tau^{\prime}$-axis in order to provide the history needed for TBCs in the current time step (represented by horizontal or vertical line in $\left(\tau, \tau^{\prime}\right)$-plane). The empty circles are only relevant for the corner points. The values of the auxiliary field at the corners are needed for the TBCs satisfied by the auxiliary fields; this relationship is depicted by the broken lines. Note that these values at the corners can be taken from the adjacent segment of the boundary where it is already being computed.

Remark II.2. From the discussion above, we obtain the following useful definition of the operator $\left(\partial_{t}-i \partial_{x}^{2}\right)^{1 / 2}$ :

$$
\left(\partial_{t}-i \partial_{x}^{2}\right)^{1 / 2} f(x, t)=\frac{1}{2 \pi} \iint d \zeta d x^{\prime} e^{-i \zeta\left(x-x^{\prime}\right)}\left[\partial_{t^{\prime}}^{1 / 2} e^{-i \zeta^{2}\left(t-t^{\prime}\right)} f\left(x^{\prime}, t^{\prime}\right)\right]_{t^{\prime}=t},
$$

for $(x, t) \in \mathbb{R} \times \mathbb{R}_{+}$. This definition makes it explicit that one has to consider the function $f(x, t)$ over its entire support with respect to $x \in \mathbb{R}$ in order to compute the expression; however, in special cases this can be avoided. Similarly, a formal definition of the operator $\left(\partial_{t}-i \partial_{x}^{2}\right)^{-m / 2},(x, t) \in \mathbb{R} \times \mathbb{R}_{+}, m>$ 0 , for any arbitrary function $f(x, t)$ with sufficient smoothness property can be given. This operator can be defined as

$$
\left(\partial_{t}-i \partial_{x}^{2}\right)^{-m / 2} f(x, t)=\frac{1}{2 \pi} \iint d \zeta d x^{\prime} e^{-i \zeta\left(x-x^{\prime}\right)}\left[\partial_{t^{\prime}}^{-m / 2} e^{-i \zeta^{2}\left(t-t^{\prime}\right)} f\left(x^{\prime}, t^{\prime}\right)\right]_{t^{\prime}=t} .
$$

The integration with respect to $\zeta$ can be easily performed. Defining the convolution kernel $\mathcal{K}(x, t)$ by

$$
\mathcal{K}(x, t)= \begin{cases}\frac{e^{-i \pi / 4}}{2 \sqrt{\pi} \Gamma(m / 2)} t^{-\frac{m+3}{2}} \exp \left[i \frac{x^{2}}{4 t}\right], & t>0, \\ 0, & t<0,\end{cases}
$$

we obtain

$$
\left(\partial_{t}-i \partial_{x}^{2}\right)^{-m / 2} f(x, t)=\iint d x^{\prime} d t^{\prime} \mathcal{K}\left(x-x^{\prime}, t-t^{\prime}\right) f\left(x^{\prime}, t^{\prime}\right)
$$

From this definition, it is clear that the operator cannot be defined on a compact domain with respect to $x$ for arbitrary functions. Let us introduce $\varphi\left(x, t, t^{\prime}\right)$ defined by

so that

$$
\varphi\left(x, t, t^{\prime}\right)=\frac{1}{2 \pi} \iint d \zeta d x^{\prime} e^{-i \zeta\left(x-x^{\prime}\right)} e^{-i \zeta^{2}\left(t-t^{\prime}\right)} f\left(x^{\prime}, t^{\prime}\right)
$$

$$
\left(\partial_{t}-i \partial_{x}^{2}\right)^{-m / 2} f(x, t)=\left.\partial_{t^{\prime}}^{-m / 2} \varphi\left(x, t, t^{\prime}\right)\right|_{t^{\prime}=t},
$$

where the auxiliary field satisfies the IVP given by

$$
\begin{aligned}
& i \partial_{\tau} \varphi\left(x, \tau, \tau^{\prime}\right)+\partial_{x}^{2} \varphi\left(x, \tau, \tau^{\prime}\right)=0, \quad \tau \in\left(\tau^{\prime}, t\right], \\
& \varphi\left(x, \tau^{\prime}, \tau^{\prime}\right)=f\left(x, \tau^{\prime}\right) .
\end{aligned}
$$

Again, the solution of the IVP must be obtained for all $\tau^{\prime} \in[0, t]$. If $f(x, \tau)$ has a bounded support, $\Omega$, with respect to $x$ for all $\tau \in[0, t]$, then the IVP above can be solved under the Dirichlet boundary condition on $\partial \Omega$ (which is not a significantly different situation that the periodic case discussed in Remark II.1) 


\section{High-frequency approximation}

The high-frequency approximation affords the possibility of simplifying the TBC by making them "local" in terms of the spatial variable. The asymptotic expansion worked out in (19) can be carried out for each of the edges of the rectangular domain to obtain the following ABCs:

$$
\begin{array}{ll}
\partial_{n} u+e^{-i \pi / 4} \partial_{t}^{1 / 2} u-e^{i \pi / 4} \frac{1}{2} \partial_{x_{2}}^{2} \partial_{t}^{-1 / 2} u=0, & \mathbf{x} \in \Gamma_{r} \cup \Gamma_{l}, \\
\partial_{n} u+e^{-i \pi / 4} \partial_{t}^{1 / 2} u-e^{i \pi / 4} \frac{1}{2} \partial_{x_{1}}^{2} \partial_{t}^{-1 / 2} u=0, & \mathbf{x} \in \Gamma_{b} \cup \Gamma_{t} .
\end{array}
$$

These boundary conditions become problematic at the corners of the rectangular domain. This aspect can be illustrated by the considering the weak formulation of the original IVP as follows: Consider a test function $\psi(\mathbf{x}) \in \mathrm{W}^{1,2}\left(\Omega_{i}\right)$ (Sobolev space); taking the inner product with the equation (6), we have

$$
\int_{\Omega_{i}}\left(i \partial_{t} u+\nabla^{2} u\right) \psi d^{2} \mathbf{x}=i \partial_{t} \int_{\Omega_{i}} u \psi d^{2} \mathbf{x}-\int_{\Omega_{i}}(\nabla u) \cdot(\nabla \psi) d^{2} \mathbf{x}+\int_{\partial \Omega_{i}} \psi(\nabla u) \cdot d \boldsymbol{\varsigma} .
$$

Let us consider the top and right boundaries. The boundary integrals are given by

$$
\begin{aligned}
& \int_{\Gamma_{r}} \psi \partial_{x_{1}} u d x_{2}+\int_{\Gamma_{t}} \psi \partial_{x_{2}} u d x_{1} \\
& =-e^{-i \pi / 4} \int_{\Gamma_{r} \cup \Gamma_{l}} \psi \partial_{t}^{1 / 2} u+\frac{1}{2} e^{i \pi / 4}\left[\int_{\Gamma_{r}} \psi \partial_{x_{2}}^{2} \partial_{t}^{-1 / 2} u d x_{2}+\int_{\Gamma_{t}} \psi \partial_{x_{1}}^{2} \partial_{t}^{-1 / 2} u d x_{1}\right] \\
& =-e^{-i \pi / 4} \int_{\Gamma_{r} \cup \Gamma_{l}} \psi \partial_{t}^{1 / 2} u+\frac{1}{2} e^{i \pi / 4}\left[\left.\psi \partial_{x_{2}} \partial_{t}^{-1 / 2} u\right|_{x_{2}=x_{b}} ^{x_{t}}+\left.\psi \partial_{x_{1}} \partial_{t}^{-1 / 2} u\right|_{x_{1}=x_{l}} ^{x_{r}}\right] \\
& \quad-\frac{1}{2} e^{i \pi / 4}\left[\int_{\Gamma_{r}}\left(\partial_{x_{2}} \psi\right)\left(\partial_{x_{2}} \partial_{t}^{-1 / 2} u\right) d x_{2}+\int_{\Gamma_{t}}\left(\partial_{x_{1}} \psi\right)\left(\partial_{x_{1}} \partial_{t}^{-1 / 2} u\right) d x_{1}\right] .
\end{aligned}
$$

Consider the terms which correspond to the top-right corner in the above equation:

$$
\left(\partial_{x_{2}} \partial_{t}^{-1 / 2} u+\partial_{x_{1}} \partial_{t}^{-1 / 2} u\right)_{\Gamma_{r} \cap \Gamma_{t}}=\partial_{t}^{-1 / 2}\left(\partial_{x_{2}} u+\partial_{x_{1}} u\right)_{\Gamma_{r} \cap \Gamma_{t}}
$$

They are problematic on account of the fact that the BCs in the current form cannot be used to evaluate them. In order to evaluate these terms, we carry out the fractional integration, $\partial_{t}^{-1 / 2}$, of the evolution equation in (6) so that

$$
i \partial_{t}^{1 / 2} u+\left(\partial_{x_{1}}^{2}+\partial_{x_{2}}^{2}\right) \partial_{t}^{-1 / 2} u=0,\left(x_{1}, x_{2}\right) \in \Gamma_{r} \cap \Gamma_{t} .
$$

Here, the fact that the field is zero at the corner at $t=0$ is explicitly used to arrive at the fractional derivative. Using BCs in (45) and the last equation, we obtained the following corner condition:

$$
\partial_{x_{1}} u+\partial_{x_{2}} u+\frac{3}{2} e^{-i \pi / 4} \partial_{t}^{1 / 2} u=0,\left(x_{1}, x_{2}\right) \in \Gamma_{r} \cap \Gamma_{t} .
$$

A similar procedure can be used to the construct corner conditions for the other corners of the rectangular domain:

$$
\left.\partial_{n} u\right|_{\Gamma_{i}}+\left.\partial_{n} u\right|_{\Gamma_{j}}+\frac{3}{2} e^{-i \pi / 4} \partial_{t}^{1 / 2} u=0,\left(x_{1}, x_{2}\right) \in \Gamma_{i} \cap \Gamma_{j},
$$

where $i \neq j$ and $i, j \in\{r, t, l, b\}$. 


\section{Stability and uniqueness}

Let us write the equivalent formulation of the IVP (6) for a rectangular domain $\Omega_{i}$ using the TBCs derived in the last section:

$$
\left\{\begin{array}{l}
i \partial_{t} u+\Delta u=0, \quad(\mathbf{x}, t) \in \Omega_{i} \times \mathbb{R}_{+}, \\
u(\mathbf{x}, 0)=u_{0}(\mathbf{x}) \in \mathrm{L}^{2}\left(\Omega_{i}\right), \quad \operatorname{supp} u_{0} \subset \Omega_{i}, \\
\partial_{n} u+e^{-i \pi / 4}\left(\partial_{t}-i \partial_{x_{2}}^{2}\right)^{1 / 2} u=0, \quad \mathbf{x} \in \Gamma_{l} \cup \Gamma_{r}, \\
\partial_{n} u+e^{-i \pi / 4}\left(\partial_{t}-i \partial_{x_{1}}^{2}\right)^{1 / 2} u=0, \quad \mathbf{x} \in \Gamma_{b} \cup \Gamma_{t}, t>0 .
\end{array}\right.
$$

Assuming that the solution $u(\mathbf{x}, t)$ exits for $t \in[0, T]$, we have

$$
\|u(\cdot, T)\|_{\mathrm{L}^{2}\left(\Omega_{i}\right)}^{2}-\left\|u_{0}\right\|_{\mathrm{L}^{2}\left(\Omega_{i}\right)}^{2}=2 \operatorname{Re} \int_{0}^{T} d t\left[\int_{\Gamma_{l} \cup \Gamma_{r}}\left(u^{*} i \nabla u\right) \cdot d \boldsymbol{s}+\int_{\Gamma_{b} \cup \Gamma_{t}}\left(u^{*} i \nabla u\right) \cdot d \boldsymbol{s}\right] .
$$

In the above equation, the fields in the boundary integral can be extended to $\mathbb{R}_{+}$without changing the value of the integral by setting them identically to zero outside the interval $[0, T]$. For the right boundary, we have

$$
\mathcal{I}_{R}=-2 \operatorname{Re} \int_{0}^{T} d t \int_{\Gamma_{r}}\left(u^{*} e^{i \pi / 4} \sqrt{\partial_{t}-i \partial_{x_{2}}^{2}} u\right) d x_{2}
$$

Again, without changing the value of the integral with respect to $x_{2}$, the fields can be extended to whole line by setting them identically to zero outside $\Gamma_{r}$. We these considerations in mind, one can write

$$
\begin{aligned}
\mathcal{I}_{R} & =-\frac{1}{\pi} \operatorname{Re} \int_{\mathbb{R}_{+}} d t \int_{\mathbb{R}} d \zeta_{2} \overline{\left(\tilde{u}\left(x_{r}, \zeta_{2}, t\right) e^{-i \zeta_{2}^{2} t}\right)}\left(\partial_{t}^{\frac{1}{2}} \tilde{u}\left(x_{r}, \zeta_{2}, t\right) e^{-i \zeta_{2}^{2} t}\right), \\
& =-\frac{1}{2 \pi^{2}} \int_{\mathbb{R}_{-}} d \xi \int_{\mathbb{R}^{2}} d \zeta_{2}|\xi|^{\frac{1}{2}}\left|\mathscr{F}_{t}\left[\tilde{u}\left(x_{r}, \zeta_{2}, t\right) e^{-i \zeta_{2}^{2} t}\right]\left(x_{r}, \zeta_{2}, \xi\right)\right|^{2}
\end{aligned}
$$

so that $\mathcal{I}_{R} \leq 0$. Similarly, it can be shown that the other boundary integrals namely $\mathcal{I}_{L}, \mathcal{I}_{B}$ and $\mathcal{I}_{T}$ corresponding to the left, bottom and top boundary, respectively, also satisfy the same inequality as that of $\mathcal{I}_{R}$. Therefore, we have

$$
\|u(\cdot, T)\|_{\mathrm{L}^{2}\left(\Omega_{i}\right)} \leq\left\|u_{0}\right\|_{\mathrm{L}^{2}\left(\Omega_{i}\right)} .
$$

Since $T \in \mathbb{R}_{+}$is arbitrary, one replace $T$ with $t$ in the above inequality. This result also guarantees the uniqueness of the solution.

For the high-frequency approximation, we have the following equivalent formulation on $\Omega_{i}$ : Putting $\Gamma=\partial \Omega_{i}$ and $\Gamma_{C}$, the set of four corner points, denote the Laplace-Beltrami operator by $\Delta_{\Gamma}$ so that

$$
\left\{\begin{array}{l}
i \partial_{t} u+\Delta u=0, \quad(\mathbf{x}, t) \in \Omega_{i} \times \mathbb{R}_{+}, \\
u(\mathbf{x}, 0)=u_{0}(\mathbf{x}) \in \mathrm{L}^{2}\left(\Omega_{i}\right), \quad \text { supp } u_{0} \subset \Omega_{i}, \\
\partial_{n} u+e^{-i \pi / 4} \partial_{t}^{1 / 2} u-e^{i \pi / 4} \frac{1}{2} \triangle_{\Gamma} \partial_{t}^{-\frac{1}{2}} u=0, \quad \mathbf{x} \in \Gamma \backslash \Gamma_{C}, \\
\left.\partial_{n} u\right|_{\Gamma_{i}}+\left.\partial_{n} u\right|_{\Gamma_{j}}+\frac{3}{2} e^{-i \pi / 4} \partial_{t}^{\frac{1}{2}} u=0, \mathbf{x} \in \Gamma_{i} \cap \Gamma_{j}, t>0,
\end{array}\right.
$$

where $i \neq j$, and $i, j \in\{r, t, l, b\}$. Taking equation (51) and following the standard approach, we have

$$
\begin{aligned}
\int_{\Gamma}\left(u^{*} i \nabla u\right) \cdot d \boldsymbol{s}= & -e^{i \pi / 4} \int_{\Gamma} u^{*} \partial_{t}^{\frac{1}{2}} u|d \mathbf{x}|-\frac{1}{2} e^{-i \pi / 4} \int_{\Gamma \backslash \Gamma_{C}} u^{*}\left(\triangle_{\Gamma} \partial_{t}^{-\frac{1}{2}} u\right)|d \mathbf{x}| \\
=- & e^{i \pi / 4} \int_{\Gamma} u^{*} \partial_{t}^{\frac{1}{2}} u|d \mathbf{x}|+\frac{1}{2} e^{-i \pi / 4} \int_{\Gamma \backslash \Gamma_{C}}\left(\partial_{\Gamma} u\right)^{*}\left(\partial_{\Gamma} \partial_{t}^{-\frac{1}{2}} u\right)|d \mathbf{x}| \\
& +\frac{3}{4} i\left(\left|u\left(x_{r}, x_{t}\right)\right|^{2}-\left|u\left(x_{l}, x_{t}\right)\right|^{2}+\left|u\left(x_{l}, x_{b}\right)\right|^{2}-\left|u\left(x_{r}, x_{b}\right)\right|^{2}\right) .
\end{aligned}
$$

Taking the real part and plugging the result back into equation (51) yields 


$$
\begin{aligned}
\|u(\cdot, T)\|_{\mathrm{L}^{2}\left(\Omega_{i}\right)}^{2}-\left\|u_{0}\right\|_{\mathrm{L}^{2}\left(\Omega_{i}\right)}^{2} & =2 \operatorname{Re} \int_{0}^{T} d t\left[-e^{i \pi / 4} \int_{\Gamma} u^{*} \partial_{t}^{1 / 2} u|d \mathbf{x}|+\frac{1}{2} e^{-i \pi / 4} \int_{\Gamma \backslash \Gamma_{C}}\left(\partial_{\Gamma} u\right)^{*}\left(\partial_{\Gamma} \partial_{t}^{-1 / 2} u\right)|d \mathbf{x}|\right] \\
& =2 \int_{\mathbb{R}_{-}} d \xi \sum_{\Gamma_{i}} \int_{\mathbb{R}} d \zeta\left(-|\xi|^{1 / 2}+\frac{1}{2}|\xi|^{-1 / 2}|\zeta|^{2}\right)\left|\mathscr{F}_{t} \mathscr{F}_{\varsigma}[u(\mathbf{x}, t)]\right|^{2},
\end{aligned}
$$

where $\varsigma$ is taken as the tangential variable to the boundary so that $\partial_{\Gamma}=\partial_{\varsigma}$. In the region defined by $|\xi| \geq|\zeta|^{2} / 2$, i.e., under the high-frequency approximation, we obtain

$$
\|u(\cdot, T)\|_{L^{2}\left(\Omega_{i}\right)} \leq\left\|u_{0}\right\|_{L^{2}\left(\Omega_{i}\right)} .
$$

\section{GENERAL SCHRÖDINGER EQUATION}

Let us consider the IVP corresponding to the general Schrödinger equation given by

$$
\begin{aligned}
& i \partial_{t} u+\Delta u+\phi\left(\mathbf{x}, t,|u|^{2}\right) u=0, \quad(\mathbf{x}, t) \in \mathbb{R}^{2} \times \mathbb{R}_{+}, \\
& u(\mathbf{x}, 0)=u_{0}(\mathbf{x}), \quad \mathbf{x} \in \mathbb{R}^{2} .
\end{aligned}
$$

The potential function, $\phi$, is assumed to be real valued. Define

$$
\Phi=\int_{0}^{t} \phi\left(\mathbf{x}, t^{\prime},\left|u\left(\mathbf{x}, t^{\prime}\right)\right|^{2}\right) d t^{\prime}
$$

The linear Schrödinger equation with time-dependent potential and the nonlinear case are treated in the same fashion in this section.

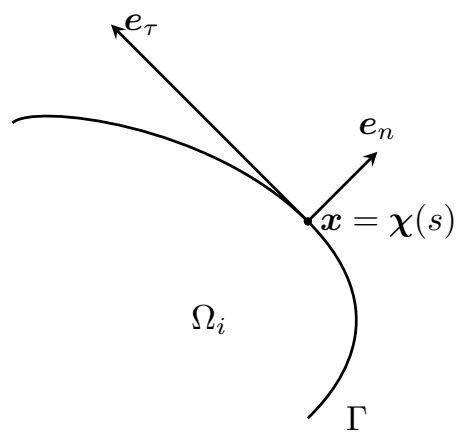

FIG. 5. The figure shows the parametrization of the boundary $\Gamma$ of a convex domain $\Omega_{i}$. Here, $\mathbf{e}_{n}$ and $\mathbf{e}_{\tau}$ denote the normal and the tangent vectors, respectively.

\section{A. Convex domain with smooth boundary}

The computational domain is taken to be a convex set $\Omega_{i} \subset \mathbb{R}^{2}$ with a smooth boundary $\Gamma=\partial \Omega_{i}$. Let $\chi(s)$ be the parametrization of the curve $\Gamma$ where ' $s$ ' is the length along the curve. Introducing the tangent vector $\mathbf{e}_{\tau}$, also a function of $s$ only, and taking into account the convexity of the domain we have

$$
d \mathbf{x}=d r \mathbf{e}_{n}+(1+r \kappa) d s \mathbf{e}_{\tau},
$$


where $\kappa$ is the curvature of the boundary $\Gamma$ given by

$$
\kappa(s)=\left|\frac{d \mathbf{e}_{\tau}}{d s}\right|=\frac{\left|\operatorname{det}\left(\chi^{\prime}, \chi^{\prime \prime}\right)\right|}{\left|\chi^{\prime}\right|^{3}},
$$

and $h=(1+r \kappa)$. The initial data is assumed to be compactly supported in the computational domain $\Omega_{i}$. Carrying out the gauge transformation $u=v e^{i \Phi}$ where $\Phi$ is defined by (55), the evolution operator $L \equiv i \partial_{t}+\Delta+\phi$ in the curvilinear system is given by

$$
L\left(r, s, t, \partial_{r}, \partial_{s}, \partial_{t}\right)=i \partial_{t}+\partial_{r}^{2}+A \partial_{r}+h^{-2} \partial_{s}^{2}+B \partial_{s}+C
$$

where $A=\left(2 i \Phi_{r}+h^{-1} \kappa\right), B=\left(2 i h^{-2} \Phi_{s}+h^{-1} \partial_{s} h^{-1}\right)$ and $C=i \triangle \Phi-(\nabla \Phi)^{2}$. The pseudo-differential approach, which uses a Nirenberg-type factorization ${ }^{24}$, allows us to construct various order ABCs given $b^{3,6}$

$$
\begin{array}{ll}
\mathrm{ABC}_{1 a}: & \partial_{n} u+e^{-i \pi / 4} e^{i \Phi}\left(\partial_{t}-i \partial_{s}^{2}\right)^{1 / 2} e^{-i \Phi} u=0 ; \\
\mathrm{ABC}_{2 a}: & \partial_{n} u+e^{-i \pi / 4} e^{i \Phi}\left(\partial_{t}-i \partial_{s}^{2}\right)^{1 / 2} e^{-i \Phi} u \\
& +\frac{1}{2} \kappa u+e^{-i \pi / 4} \Phi_{s} e^{i \Phi}\left(\partial_{t}-i \partial_{s}^{2}\right)^{-1 / 2} \partial_{s}\left(e^{-i \Phi} u\right)+\frac{1}{2} i \kappa e^{i \Phi}\left(\partial_{t}-i \partial_{s}^{2}\right)^{-1} \partial_{s}^{2}\left(e^{-i \Phi} u\right)=0,
\end{array}
$$

where

$$
\left(\partial_{t}-i \partial_{s}^{2}\right)^{1 / 2} f(s, t)=\left(\partial_{t}-i \partial_{s}^{2}\right)\left(\partial_{t}-i \partial_{s}^{2}\right)^{-1 / 2} f(s, t),
$$

assuming $f(s, 0)=0$.

Remark III.1. For the field $u(\mathbf{x}, t)$, the action of operators of the form $\left(\partial_{t}-i \partial_{s}^{2}\right)^{\alpha}, \alpha=1 / 2,-1 / 2,-1, \ldots$, can be easily computed by observing that the field can be given a periodic extension in terms of the parametrization variable, s, so that the method discussed in Remark II.1 can be employed. The smoothness of the boundary is, therefore, a necessary condition for this method to be applicable. From the previous sections, we know that the operator represented by $\left(\partial_{t}-i \partial_{s}^{2}\right)^{-m / 2}$, where $m$ is a positive integer, is defined as

$$
\left(\partial_{t}-i \partial_{s}^{2}\right)^{-m / 2} f(s, t)=\frac{1}{2 \pi} \iint d \zeta d s^{\prime} e^{i \zeta\left(s-s^{\prime}\right)}\left[\partial_{t^{\prime}}^{-m / 2} e^{-i \zeta^{2}\left(t-t^{\prime}\right)} f\left(s^{\prime}, t^{\prime}\right)\right]_{t^{\prime}=t} .
$$

Let $\mathscr{F}_{s} f(s, t)=\tilde{f}(\zeta, t)$, then the operation $\left(\partial_{t}-i \partial_{s}^{2}\right)^{-m / 2}$ involves the inverse Fourier transform of $\tilde{f}\left(\zeta, t^{\prime}\right) e^{-i \zeta^{2}\left(t-t^{\prime}\right)}$ with respect to $\zeta$. Therefore, if $f(s, t)$ is of the Schwartz class (with respect to $s \in \mathbb{R}$ ) then so is $\tilde{f}\left(\zeta, t^{\prime}\right) e^{-i \zeta^{2}\left(t-t^{\prime}\right)}$, so that $\left(\partial_{t}-i \partial_{s}^{2}\right)^{-m / 2} f(s, t)$ will also be of the Schwartz class (with respect to $s \in \mathbb{R})$. Further, it is straightforward to show that $\left(\partial_{t}-i \partial_{s}^{2}\right)^{-m / 2} f(s, t)$ is continuous at $t=0$.

Next, our aim is to define the operator $\left(\partial_{t}-i \partial_{s}^{2}\right)^{-m / 2}$ for tempered distributions $f(s, t)$ such that $\operatorname{supp}_{t} f \subset[0, \infty)$. To this end, let us observe that the transpose $\left[\left(\partial_{t}-i \partial_{s}^{2}\right)^{-m / 2}\right]^{\top}$ is given by

$$
\left[\left(\partial_{t}-i \partial_{s}^{2}\right)^{-m / 2}\right]^{\top} g(s, t)=\frac{1}{2 \pi} \iint d \zeta d s^{\prime} e^{i \zeta\left(s-s^{\prime}\right)}\left[\left(\partial_{t^{\prime}}^{-m / 2}\right)^{\top} e^{-i \zeta^{2}\left(t-t^{\prime}\right)} g\left(s^{\prime}, t^{\prime}\right)\right]_{t^{\prime}=t},
$$

where $\left(\partial_{t^{\prime}}^{-m / 2}\right)^{\top}$ denotes the Weyl fractional integral. The domain of definition of this operator is evident. Now, for any $g(s, t) \in \mathrm{S}(\mathbb{R} \times \mathbb{R})$ with $\operatorname{supp}_{t} g \subset[0, \infty)$, the operation $\left(\partial_{t}-i \partial_{s}^{2}\right)^{-m / 2} f(s, t)$ for distributions $f(s, t) \in \mathrm{S}^{\prime}(\mathbb{R} \times \mathbb{R})$ with supp $f \subset[0, \infty)$ can be defined by introducing a Schwartz class function $g_{1}(s, t)$ such that it agrees with $\left[\left(\partial_{t}-i \partial_{s}^{2}\right)^{-m / 2}\right]^{\top} g(s, t)$ for $t \in[0, \infty)$ so that

$$
\left\langle g,\left(\partial_{t}-i \partial_{s}^{2}\right)^{-m / 2} f\right\rangle=\left\langle\left[\left(\partial_{t}-i \partial_{s}^{2}\right)^{-m / 2}\right]^{\top} g, f\right\rangle=\left\langle g_{1}, f\right\rangle .
$$

Therefore, $\left(\partial_{t}-i \partial_{s}^{2}\right)^{-m / 2} f(s, t)$ is also tempered such that $\operatorname{supp}_{t}\left(\partial_{t}-i \partial_{s}^{2}\right)^{-m / 2} f(s, t) \subset[0, \infty)$. Note that any function of the tangential variable, $s$, can be extended periodically on the whole line. Thus, periodic functions being a tempered distribution can be easily included in the domain of definition of $\left(\partial_{t}-i \partial_{s}^{2}\right)^{-m / 2}$. It is interesting to observe that by applying the Leibniz formula for the fractional integrals one can obtain local approximation of the ABCs with respect to s: 


$$
\begin{aligned}
& \left(\partial_{t}-i \partial_{s}^{2}\right)^{-m / 2} f(s, t)=\frac{1}{2 \pi} \iint d \zeta d s^{\prime} e^{i \zeta\left(s-s^{\prime}\right)}\left[\partial_{t^{\prime}}^{-m / 2} e^{-i \zeta^{2}\left(t-t^{\prime}\right)} f\left(s^{\prime}, t^{\prime}\right)\right]_{t^{\prime}=t} \\
& =\frac{1}{2 \pi} \iint d \zeta d s^{\prime} e^{i \zeta\left(s-s^{\prime}\right)}\left[\sum_{j \in \mathbb{N}}\left(\begin{array}{c}
-m / 2 \\
j
\end{array}\right)\left(\partial_{t^{\prime}}^{j} e^{-i \zeta^{2}\left(t-t^{\prime}\right)}\right) \partial_{t^{\prime}}^{-m / 2-j} f\left(s^{\prime}, t^{\prime}\right)\right]_{t^{\prime}=t} \\
& =\frac{1}{2 \pi} \iint d \zeta d s^{\prime} e^{i \zeta\left(s-s^{\prime}\right)}\left[\sum_{j \in \mathbb{N}}\left(\begin{array}{c}
-m / 2 \\
j
\end{array}\right)\left(-i \zeta^{2}\right)^{j} \partial_{t}^{-m / 2-j} f\left(s^{\prime}, t\right)\right] \\
& =\sum_{j \in \mathbb{N}}\left(\begin{array}{c}
-m / 2 \\
j
\end{array}\right)\left(i \partial_{s}^{2}\right)^{j} \partial_{t}^{-m / 2-j} f(s, t) .
\end{aligned}
$$

The second family of various order ABCs are obtained under high-frequency assumption with respect to the temporal frequency. These are given by

$$
\begin{array}{cc}
\mathrm{ABC}_{1 b}: & \partial_{n} u+e^{-i \pi / 4} e^{i \Phi} \partial_{t}^{1 / 2} e^{-i \Phi} u=0 \\
\mathrm{ABC}_{2 b}: & \partial_{n} u+e^{-i \pi / 4} e^{i \Phi} \partial_{t}^{1 / 2} e^{-i \Phi} u+\frac{1}{2} \kappa u-\frac{1}{2} e^{i \pi / 4}\left(\frac{\kappa^{2}}{4}+\partial_{s}^{2}\right) e^{i \Phi} \partial_{t}^{-1 / 2} e^{-i \Phi} u=0 ; \\
\mathrm{ABC}_{3 b}: & \partial_{n} u+e^{-i \pi / 4} e^{i \Phi} \partial_{t}^{1 / 2} e^{-i \Phi} u+\frac{1}{2} \kappa u-\frac{1}{2} e^{i \pi / 4}\left(\frac{\kappa^{2}}{4}+\partial_{s}^{2}\right) e^{i \Phi} \partial_{t}^{-1 / 2} e^{-i \Phi} u \\
+ & \frac{i}{2}\left[\frac{\kappa^{3}+\partial_{s}^{2} \kappa}{4}-\frac{\partial_{n} \phi}{2}+\partial_{s}\left(\kappa \partial_{s}\right)\right] e^{i \Phi} \partial_{t}^{-1} e^{-i \Phi} u=0 .
\end{array}
$$

\section{Stability and uniqueness}

In order to study the stability property of the solution of the IVP defined by (54) with boundary condition $\mathrm{ABC}_{1 a}$ and $\mathrm{ABC}_{2 a}$, respectively, we start with the relation:

$$
\|u(\cdot, T)\|_{\mathrm{L}^{2}\left(\Omega_{i}\right)}^{2}-\left\|u_{0}\right\|_{\mathrm{L}^{2}\left(\Omega_{i}\right)}^{2}=2 \operatorname{Re} \int_{0}^{T} d t \int_{\Gamma}\left(u^{*} i \nabla u\right) \cdot d \boldsymbol{\varsigma} .
$$

Realization of the boundary operators require an appropriate Fourier representation with respect to the tangential variable, $s$. To this end, one may either employ the Fourier series representation by extending the field periodically for all $s \in \mathbb{R}$ or Fourier transform representation by extending the field to all $s \in \mathbb{R}$ by setting it zero outside $\Gamma$. The result for the first order $\mathrm{ABCs}, \mathrm{ABC}_{1 a}$, can be obtained by observing that the boundary integral

$$
\mathcal{I}_{1}=-e^{i \pi / 4} \int_{0}^{T} d t \int_{\Gamma} \overline{\left(u e^{-i \Phi}\right)} \sqrt{\partial_{t}-i \partial_{s}^{2}}\left(u e^{-i \Phi}\right) d s,
$$

satisfies $\operatorname{Re} \mathcal{I}_{1} \leq 0$ so that

$$
\|u(\cdot, T)\|_{L^{2}\left(\Omega_{i}\right)} \leq\left\|u_{0}\right\|_{L^{2}\left(\Omega_{i}\right)} .
$$

For the second order $\mathrm{ABCs}, \mathrm{ABC}_{2 a}$, the energy estimate cannot be obtained for a general potential function. A special case of interest is when $\partial_{s} \phi=0$ so that $\mathrm{ABC}_{2 a}$ is given by

$$
\partial_{n} u+e^{-i \pi / 4} e^{i \Phi}\left(\partial_{t}-i \partial_{s}^{2}\right)^{1 / 2} e^{-i \Phi} u+\frac{1}{2} \kappa u+\frac{1}{2} i \kappa e^{i \Phi}\left(\partial_{t}-i \partial_{s}^{2}\right)^{-1} \partial_{s}^{2}\left(e^{-i \Phi} u\right)=0
$$

Define the boundary integrals

$$
\begin{aligned}
& \mathcal{I}_{2}=-\frac{i}{2} \int_{0}^{T} d t \int_{\Gamma} \kappa(s)|u|^{2} d s, \\
& \mathcal{I}_{3}=\frac{1}{2} \int_{0}^{T} d t \int_{\Gamma} \kappa(s) \overline{\left(u e^{-i \Phi}\right)}\left(\partial_{t}-i \partial_{s}^{2}\right)^{-1} \partial_{s}^{2}\left(u e^{-i \Phi}\right) d s .
\end{aligned}
$$


It follows that $\operatorname{Re} \mathcal{I}_{2}=0$. Define the symbol $\sigma_{P}=(1 / 2) \kappa(s)\left(i \xi+i \zeta^{2}\right)^{-1}\left(-\zeta^{2}\right)$ so that the symbol for the adjoint operator $\sigma_{P^{\dagger}}$ works out to be

$$
\sigma_{P^{\dagger}} \sim \frac{-i}{2} \sum_{k \in \mathbb{N}} \frac{1}{k ! i^{k}}\left(\partial_{s}^{k} \kappa\right) \partial_{\zeta}^{k}\left(\frac{\zeta^{2}}{\xi+\zeta^{2}}\right) .
$$

Let $2 Q=P+P^{\dagger}$ so that

$$
\begin{aligned}
2 \sigma_{Q} & \sim \frac{i}{2} \frac{\kappa \zeta^{2}}{\xi+\zeta^{2}}+\frac{-i}{2} \sum_{k \in \mathbb{N}} \frac{1}{k ! i^{k}}\left(\partial_{s}^{k} \kappa\right) \partial_{\zeta}^{k}\left(\frac{\zeta^{2}}{\xi+\zeta^{2}}\right) \\
& =-\frac{1}{2}\left(\partial_{s} \kappa\right) \partial_{\zeta}\left(\frac{\zeta^{2}}{\xi+\zeta^{2}}\right)+\ldots,
\end{aligned}
$$

and putting $\psi=u e^{-i \Phi}$, we have

$$
\operatorname{Re} \mathcal{I}_{3}=\frac{1}{(2 \pi)^{2}} \iiint d \zeta d \xi d s \mathscr{F}_{t} \mathscr{F}_{s}[\psi(s, t)] \sigma_{Q}(s, \zeta, \xi) \mathscr{F}_{t}[\psi(s, t)] e^{-i \zeta s} .
$$

From the asymptotic expansion, to the zeroth order, we have $\operatorname{Re} \mathcal{I}_{3} \approx 0$ (for constant curvature boundary, this result is exact). The energy estimate can thus be approximately established for the special case when $\partial_{s} \phi=0$.

For the $\mathrm{ABCs}$ obtained in the high-frequency approximation, it is evident that the first order $\mathrm{ABC}, \mathrm{ABC}_{1 b}$, satisfies the energy estimate. For the higher-order $\mathrm{ABCs}$, one requires an additional conditions, $\partial_{s} \phi=0$ in order to obtain the energy estimate. In $\mathrm{ABC}_{2 b}$, the term with the factor $\kappa / 2$ can be ignored as it leads to a purely imaginary quantity. Putting $\psi=u e^{i \Phi}$, consider the following boundary integrals

$$
\begin{aligned}
& \mathcal{I}_{1}=-\frac{e^{i \pi / 4}}{2} \int_{0}^{T} d t \int_{\Gamma} \psi^{*}(s, t)\left[2 \partial_{t}^{\frac{1}{2}}-i \partial_{t}^{-\frac{1}{2}} \partial_{s}^{2}\right] \psi(s, t) d s \\
& \mathcal{I}_{2}=-\frac{e^{-i \pi / 4}}{8} \int_{0}^{T} d t \int_{\Gamma} \psi^{*}(s, t) \kappa^{2} \partial_{t}^{-\frac{1}{2}} \psi(s, t) d s
\end{aligned}
$$

The first integral, $\mathcal{I}_{1}$, can be dealt with in the manner done before. The second integral can be dealt with in the manner described in the appendix. Defining $\sigma_{P}=-e^{-i \pi / 4} \kappa^{2}(i \xi)^{-1 / 2}$ so that $\sigma_{P^{\dagger}}=$ $-e^{i \pi / 4} \kappa^{2}(-i \xi)^{-1 / 2}$. Putting $2 Q=P+P^{\dagger}$, we have $\sigma_{Q}=-\kappa^{2} \cos [\pi / 4+\pi \operatorname{sgn}(\xi) / 4]$. The boundary integral can now be written as

$$
\operatorname{Re} \mathcal{I}_{2}=-\frac{1}{16 \pi} \iint d \xi d s[\kappa(s)]^{2}|\xi|^{-1 / 2} \cos [\pi(1+\operatorname{sgn}(\xi)) / 4]\left|\mathscr{F}_{t}[\psi(s, t)]\right|^{2},
$$

so that $\operatorname{Re} I_{2} \leq 0$.

For $\mathrm{ABC}_{3 b}$, we define the following boundary integrals

$$
\begin{aligned}
& \mathcal{I}_{3}=\frac{1}{8} \int_{0}^{T} d t \int_{\Gamma} \psi^{*}(s, t)\left[\left(\kappa^{3}+\partial_{s}^{2} \kappa\right) \partial_{t}^{-1}\right] \psi(s, t) d s, \\
& \mathcal{I}_{4}=\frac{1}{2} \int_{0}^{T} d t \int_{\Gamma} \psi^{*}(s, t)\left[\left(\partial_{s} \kappa \partial_{s}\right) \partial_{t}^{-1}\right] \psi(s, t) d s, \\
& \mathcal{I}_{5}=-\frac{1}{4} \int_{0}^{T} d t \int_{\Gamma} \psi^{*}(s, t)\left[\left(\partial_{n} \phi\right) \partial_{t}^{-1}\right] \psi(s, t) d s .
\end{aligned}
$$

Defining $\sigma_{P}=\left(\kappa^{3}+\partial_{s}^{2} \kappa\right)(i \xi)^{-1}$ so that

$$
\sigma_{Q}=\left(\kappa^{3}+\partial_{s}^{2} \kappa\right)|\xi|^{-1} \cos [\pi \operatorname{sgn}(\xi) / 2] .
$$


From the above equation, it follows that $I_{3}$ is purely imaginary. Carrying out an integration by parts in $\mathcal{I}_{4}$ with respect to $s$, we have

$$
\begin{aligned}
\mathcal{I}_{4} & =-\frac{1}{2} \int_{0}^{T} d t \int_{\Gamma}\left[\partial_{s} \psi^{*}(s, t)\right] \kappa(s) \partial_{t}^{-1}\left[\partial_{s} \psi(s, t)\right] d s \\
& =-\frac{1}{2 \pi} \int_{0}^{T} d \xi \int_{\Gamma} \kappa(s)(i \xi)^{-1}\left|\mathscr{F}_{t}\left[\partial_{s} \psi(s, t)\right]\right|^{2} d s .
\end{aligned}
$$

Therefore, $\mathcal{I}_{4}$ is purely imaginary. The discussion of the last integral, $\mathcal{I}_{5}$, is more involved. Define the symbol $\sigma_{P}=-\left(\partial_{n} \phi\right)(i \xi)^{-1}$ so that the symbol for the adjoint operator $\sigma_{P^{\dagger}}$ works out to be

$$
\sigma_{P^{\dagger}} \sim-\sum_{k \in \mathbb{N}} \frac{1}{k ! i^{k}}\left(\partial_{t}^{k} \partial_{n} \phi\right) \partial_{\xi}^{k}(-i \xi)^{-1} .
$$

Now

$$
\begin{aligned}
2 \sigma_{Q} & \sim-\left(\partial_{n} \phi\right)(i \xi)^{-1}-\sum_{k \in \mathbb{N}} \frac{1}{k ! i^{k}}\left(\partial_{t}^{k} \partial_{n} \phi\right) \partial_{\xi}^{k}(-i \xi)^{-1} \\
& =-\left(\partial_{t} \partial_{n} \phi\right)(\xi)^{-2}+\ldots
\end{aligned}
$$

Therefore, the contribution from this last integral can be ignored on account of the lower order leading term in the above asymptotic expansion. Note that an alternative technique is to symmetrize the operator corresponding to $\mathcal{I}_{5}$ as suggested in Ref. 9:

$$
-\frac{i \operatorname{sgn}\left[\partial_{n} \phi\right]}{4} \sqrt{\left|\partial_{n} \phi\right|} e^{i \Phi} \partial_{t}^{-1}\left(\sqrt{\left|\partial_{n} \phi\right|} e^{-i \Phi} u\right)=-\frac{i \partial_{n} \phi}{4} e^{i \Phi} \partial_{t}^{-1} e^{-i \Phi} u \bmod \left(\partial_{t}^{-2}\right) .
$$

While the order of the error term is the same for both alternatives, the unsymmetrized and the symmetrized version, the numerical conditioning of the symmetrized version is superior because $\mathcal{I}_{5}$ becomes purely imaginary ${ }^{9}$. Thus the energy estimate

$$
\|u(\cdot, T)\|_{L^{2}\left(\Omega_{i}\right)} \leq\left\|u_{0}\right\|_{L^{2}\left(\Omega_{i}\right)} .
$$

can be established (at least in the "weak" sense) for the $\mathrm{ABC}$-family: $\mathrm{ABC}_{j b}, j=1,2,3$. Let us note that only the second and third order $\mathrm{ABC}$ require $\partial_{s} \phi=0$ in order to establish the energy estimate.

\section{B. Domains with straight boundary: infinite strip}

The ABCs for the straight boundary can be written by setting the curvature $\kappa$ to zero:

$$
\begin{array}{ll}
\mathrm{ABC}_{1 a}: & \partial_{n} u+e^{-i \pi / 4} e^{i \Phi}\left(\partial_{t}-i \partial_{s}^{2}\right)^{1 / 2} e^{-i \Phi} u=0 ; \\
\mathrm{ABC}_{2 a}: & \partial_{n} u+e^{-i \pi / 4} e^{i \Phi}\left(\partial_{t}-i \partial_{s}^{2}\right)^{1 / 2} e^{-i \Phi} u+e^{-i \pi / 4} \Phi_{s} e^{i \Phi}\left(\partial_{t}-i \partial_{s}^{2}\right)^{-1 / 2} \partial_{s}\left(e^{-i \Phi} u\right)=0 .
\end{array}
$$

Along the direction in which the strip extends to infinity, we impose the periodic boundary condition. Note that an exact representation of the boundary operators is possible in this case which is based on the observations made in earlier sections. The energy estimate for the first order ABCs is easy to obtain; however, for the second order $\mathrm{ABCs}$ this result is not available. The high-frequency ABCs work out to be

$$
\begin{array}{ll}
\mathrm{ABC}_{1 b}: & \partial_{n} u+e^{-i \pi / 4} e^{i \Phi} \partial_{t}^{1 / 2} e^{-i \Phi} u=0 \\
\mathrm{ABC}_{2 b}: & \partial_{n} u+e^{-i \pi / 4} e^{i \Phi} \partial_{t}^{1 / 2} e^{-i \Phi} u-\frac{1}{2} e^{i \pi / 4} \partial_{s}^{2}\left(e^{i \Phi} \partial_{t}^{-1 / 2} e^{-i \Phi} u\right)=0 \\
\mathrm{ABC}_{3 b}: & \partial_{n} u+e^{-i \pi / 4} e^{i \Phi} \partial_{t}^{1 / 2} e^{-i \Phi} u-\frac{1}{2} e^{i \pi / 4} \partial_{s}^{2}\left(e^{i \Phi} \partial_{t}^{-1 / 2} e^{-i \Phi} u\right)-\frac{i \partial_{n} \phi}{4} e^{i \Phi} \partial_{t}^{-1} e^{-i \Phi} u=0 .
\end{array}
$$

The energy estimate for the general case is not available for such ABCs. 


\section{Rectangular domains: corner conditions}

Continuing with the rectangular domain as defined in Sec. II B, let us consider the possibility of extending the results obtained in Sec. II B 1 to the nonlinear/variable potential case. We confine our attention to the high-frequency $\mathrm{ABCs}$ only. We demonstrate the possibility of constructing the corner condition at the corner defined by $\Gamma_{r} \cap \Gamma_{t}$. Consider the $\mathrm{ABC}_{2 b}$ given by

$$
\partial_{x_{1}} u+e^{-i \pi / 4} e^{i \Phi} \partial_{t}^{1 / 2} e^{-i \Phi} u-\frac{1}{2} e^{i \pi / 4} \partial_{x_{2}}^{2}\left(e^{i \Phi} \partial_{t}^{-1 / 2} e^{-i \Phi} u\right)=0, \quad \mathbf{x} \in \Gamma_{r}
$$

and

$$
\partial_{x_{2}} u+e^{-i \pi / 4} e^{i \Phi} \partial_{t}^{1 / 2} e^{-i \Phi} u-\frac{1}{2} e^{i \pi / 4} \partial_{x_{1}}^{2}\left(e^{i \Phi} \partial_{t}^{-1 / 2} e^{-i \Phi} u\right)=0, \quad \mathbf{x} \in \Gamma_{t} .
$$

Let us consider the weak formulation of the IVP given by

$$
\begin{aligned}
& i \partial_{t} u+\Delta u+\phi u=0, \quad(\mathbf{x}, t) \in \Omega_{i} \times \mathbb{R}_{+}, \\
& u(\mathbf{x}, 0)=u_{0}(\mathbf{x}), \quad \mathbf{x} \in \Omega_{i}, \quad \operatorname{supp} u_{0} \subset \Omega_{i} .
\end{aligned}
$$

Let $\psi(\mathbf{x}) \in \mathrm{W}^{1,2}\left(\Omega_{i}\right)$ be a test function so that

$$
\int_{\Omega_{i}}\left(i \partial_{t} u+\nabla^{2} u+\phi u\right) \psi d^{2} \mathbf{x}=\int_{\Omega_{i}}\left[i \partial_{t} u-(\nabla u) \cdot(\nabla \psi)+\phi u\right] d^{2} \mathbf{x}+\int_{\Gamma} \psi(\nabla u) \cdot d \boldsymbol{\varsigma} .
$$

Focusing on the top and right boundary, the boundary integrals are given by

$$
\begin{aligned}
& \int_{\Gamma_{r}} \psi\left(\partial_{x_{1}} u\right) d x_{2}+\int_{\Gamma_{t}} \psi\left(\partial_{x_{2}} u\right) d x_{1} \\
& =-e^{-i \pi / 4} \int_{\Gamma_{r} \cup \Gamma_{l}} \psi e^{i \Phi} \partial_{t}^{1 / 2} e^{-i \Phi} u+\frac{1}{2} e^{i \pi / 4}\left[\int_{\Gamma_{r}} \psi \partial_{x_{2}}^{2}\left(e^{i \Phi} \partial_{t}^{-1 / 2} e^{-i \Phi} u\right) d x_{2}+\int_{\Gamma_{t}} \psi \partial_{x_{1}}^{2}\left(e^{i \Phi} \partial_{t}^{-1 / 2} e^{-i \Phi} u\right) d x_{1}\right] \\
& =-e^{-i \pi / 4} \int_{\Gamma_{r} \cup \Gamma_{l}} \psi e^{i \Phi} \partial_{t}^{1 / 2} e^{-i \Phi} u+\frac{1}{2} e^{i \pi / 4}\left[\left.\psi \partial_{x_{2}}\left(e^{i \Phi} \partial_{t}^{-1 / 2} e^{-i \Phi} u\right)\right|_{x_{2}=x_{b}} ^{x_{t}}+\left.\psi \partial_{x_{1}}\left(e^{i \Phi} \partial_{t}^{-1 / 2} e^{-i \Phi} u\right)\right|_{x_{1}=x_{l}} ^{x_{r}}\right] \\
& -\frac{1}{2} e^{i \pi / 4}\left[\int_{\Gamma_{r}}\left(\partial_{x_{2}} \psi\right) \partial_{x_{2}}\left(e^{i \Phi} \partial_{t}^{-1 / 2} e^{-i \Phi} u\right) d x_{2}+\int_{\Gamma_{t}}\left(\partial_{x_{1}} \psi\right) \partial_{x_{1}}\left(e^{-i \Phi} \partial_{t}^{-1 / 2} e^{-i \Phi} u\right) d x_{1}\right] .
\end{aligned}
$$

From here, it is evident that the corner condition must provide the value of the following expression at $\Gamma_{r} \cap \Gamma_{t}$ :

$$
\begin{aligned}
& \partial_{x_{1}}\left(e^{i \Phi} \partial_{t}^{-1 / 2} e^{-i \Phi} u\right)+\partial_{x_{2}}\left(e^{i \Phi} \partial_{t}^{-1 / 2} e^{-i \Phi} u\right) \\
& \quad=i\left(\Phi_{x_{1}}+\Phi_{x_{2}}\right) e^{i \Phi} \partial_{t}^{-1 / 2} e^{-i \Phi} u-i e^{i \Phi} \partial_{t}^{-1 / 2}\left[\left(\Phi_{x_{1}}+\Phi_{x_{2}}\right) e^{-i \Phi} u\right]+e^{i \Phi} \partial_{t}^{-1 / 2} e^{-i \Phi}\left(\partial_{x_{1}} u+\partial_{x_{2}} u\right)
\end{aligned}
$$

For the linear case with variable potential, the last term in the above equation must be computed from a corner condition. However, for the nonlinear case, $\Phi$ is supposed to be dependent on some known field while our final intent is to restore its nonlinear dependence. In this light, the above equation cannot be made free of the derivatives of $u$ at the corner as the equation is nonlinear. Nevertheless, we may derive a condition at the corner which can be combined with the ABCs and eventually be solved by an iterative scheme. Putting $u=\psi e^{i \Phi}$ in the evolution equation (81), we have

$$
i \partial_{t} \psi+\Delta \psi+2 i(\nabla \Phi) \cdot(\nabla \psi)+\left(e^{-i \Phi} \Delta e^{i \Phi}\right) \psi=0 .
$$

Carrying out the operation $e^{i \Phi} \partial_{t}^{-1 / 2}$ on both side of the equation above, we have 


$$
\begin{aligned}
& i e^{i \Phi} \partial_{t}^{1 / 2} \psi+e^{i \Phi} \partial_{t}^{-1 / 2} \Delta \psi+2 e^{i \Phi} \partial_{t}^{-1 / 2}(i \nabla \Phi) \cdot(\nabla \psi)+e^{i \Phi} \partial_{t}^{-1 / 2}\left(e^{-i \Phi} \Delta e^{i \Phi}\right) \psi=0 \\
& i e^{i \Phi} \partial_{t}^{1 / 2} \psi+e^{i \Phi} \partial_{t}^{-1 / 2} \Delta \psi+2 e^{i \Phi}(i \nabla \Phi) \cdot \partial_{t}^{-1 / 2}(\nabla \psi)+\left(\Delta e^{i \Phi}\right) \partial_{t}^{-1 / 2} \psi+\ldots=0
\end{aligned}
$$

which simplifies to

$$
i e^{i \Phi} \partial_{t}^{1 / 2} e^{-i \Phi} u+\Delta\left(e^{i \Phi} \partial_{t}^{-1 / 2} e^{-i \Phi} u\right)=0 \quad \bmod \left(\partial_{t}^{-3 / 2} e^{-i \Phi} u\right) .
$$

This equation combined with the ABCs gives the following corner condition

$$
\mathrm{CC}_{1}: \partial_{x_{1}} u+\partial_{x_{2}} u+\frac{3}{2} e^{-i \pi / 4} e^{i \Phi} \partial_{t}^{1 / 2} e^{-i \Phi} u=0,
$$

or, equivalently,

$$
\mathrm{CC}_{1}: e^{i \Phi} \partial_{t}^{-1 / 2} e^{-i \Phi}\left(\partial_{x_{1}} u+\partial_{x_{2}} u\right)+\frac{3}{2} e^{-i \pi / 4} u=0
$$

Similarly, for $\mathrm{ABC}_{3 b}$ one has the following corner condition

$$
\mathrm{CC}_{2}: \partial_{x_{1}} u+\partial_{x_{2}} u+\frac{3}{2} e^{-i \pi / 4} e^{i \Phi} \partial_{t}^{1 / 2} e^{-i \Phi} u-i \frac{\partial_{x_{1}} \phi+\partial_{x_{2}} \phi}{4} e^{i \Phi} \partial_{t}^{-1} e^{-i \Phi} u=0 \quad \bmod \left(\partial_{t}^{-5 / 2} e^{-i \Phi} u\right),
$$

or, equivalently,

$$
\mathrm{CC}_{2}: e^{i \Phi} \partial_{t}^{-1 / 2} e^{-i \Phi}\left(\partial_{x_{1}} u+\partial_{x_{2}} u\right)+\frac{3}{2} e^{-i \pi / 4} u-i \frac{\partial_{x_{1}} \phi+\partial_{x_{2}} \phi}{4} e^{i \Phi} \partial_{t}^{-3 / 2} e^{-i \Phi} u=0 \quad \bmod \left(\partial_{t}^{-5 / 2} e^{-i \Phi} u\right) .
$$

\section{Special case: $\phi=\phi(t)$}

For time-dependent potentials with no spatial variation, the quantity $\Phi$ is purely time-dependent and the $\mathrm{ABCs}$ for different domains types can be simplified considerably. It must be remarked that the exact form of the transparent boundary condition is only obtainable for an infinite-strip with periodic boundary condition in the unbounded direction or rectangular domains. At the appropriate segments of the boundary, it reads as

$$
\partial_{n} u+e^{-i \pi / 4} e^{i \Phi}\left(\partial_{t}-i \partial_{s}^{2}\right)^{1 / 2} e^{-i \Phi} u=0 .
$$

For rectangular domains, we consider this problem in more detail. At the boundary $\Gamma_{r}$, we have

$$
\partial_{x_{1}} u+e^{-i \pi / 4} e^{i \Phi}\left(\partial_{t}-i \partial_{x_{2}}^{2}\right)^{1 / 2} e^{-i \Phi} u=0
$$

The auxiliary function, $\varphi\left(x_{1}, x_{2}, t, t^{\prime}\right)$ as defined in Sec. II B in the present case is given by

$$
\mathscr{F}_{x_{2}}\left[\varphi\left(x_{1}, x_{2}, t, t^{\prime}\right)\right]=e^{-i \zeta_{2}^{2}\left(t-t^{\prime}\right)-i \Phi\left(t^{\prime}\right)} \mathscr{F}_{x_{2}}\left[u\left(x_{1}, x_{2}, t^{\prime}\right)\right],
$$

so that $\varphi\left(x_{1}, x_{2}, t, t\right)=u\left(x_{1}, x_{2}, t\right) e^{-i \Phi(t)}$. Putting $\tilde{u}_{0}(\zeta)=\mathscr{F}_{\left(x_{1}, x_{2}\right)} u_{0}(\mathbf{x})$, we have

$$
\begin{aligned}
& \mathscr{F}_{\left(x_{1}, x_{2}\right)} u\left(\mathbf{x}, t^{\prime}\right)=e^{-i\left(\zeta_{1}^{2}+\zeta_{2}^{2}\right) t^{\prime}+i \Phi\left(t^{\prime}\right)} \tilde{u}_{0}(\zeta), \\
& \varphi\left(x_{1}, x_{2}, t, t^{\prime}\right)=\frac{1}{(2 \pi)^{2}} \int_{\mathbb{R}^{2}} e^{i \zeta \cdot \mathbf{x}-i \zeta_{1}^{2} t^{\prime}-i \zeta_{2}^{2} t} \tilde{u}_{0}(\zeta) d^{2} \zeta
\end{aligned}
$$

From here is follows that the IVPs satisfied by this auxiliary field is not different from that described in Sec. II B. 


\section{CONCLUSION}

In this paper, we have discussed the formulation of the operator $\left(\partial_{t}-i \triangle_{\Gamma}\right)^{\alpha}, \alpha=1 / 2,-1 / 2,-1, \ldots$, in terms of the fractional operators in various settings. This allows the TBCs/ABCs for the free Schrödinger equation and general Schrödinger equation formulated on various types of computational domains to be expressed in a natural way. In particular, two families of ABCs within the gauge transformation strategy are studied in this paper: the ABCs obtained with the pseudo-differential approach and those obtained as a high-frequency approximation of the former. For the rectangular domains, we have developed various order corner conditions for the family of ABCs obtained in the high-frequency approximation. Each of these families of ABCs (along with corner conditions) are also investigated for stability and uniqueness of the solution of the resulting initial-boundary value problem. Further, we expect that the results presented in this paper can be easily extended to the pseudo-differential approach within the direct strategy ${ }^{9}$. Finally, let us remark that the ABCs obtained in this article can be readily discretized using the convolution quadrature. These issues will be addressed in a forthcoming paper.

\section{Appendix A: Some properties of the pseudo-differential operators}

Let us consider the symbol space $\mathrm{S}_{M}^{m}\left(Y \times \mathbb{R}^{n}\right)$ of $M$-quasi homogeneous symbols ${ }^{25}$ where $Y$ is an open subset of $\mathbb{R}^{n}$ and $M=\left(\mu_{1}, \mu_{2}, \ldots, \mu_{n}\right)$ is an n-tuple of numbers $\mu_{i}>0$. Let $P$ be a pseudodifferential operator with the symbol $p(y, \zeta) \in \mathrm{S}_{M}^{m}\left(Y \times \mathbb{R}^{n}\right)$ so that

$$
\begin{aligned}
P u(y) & =\int p(y, \zeta) e^{i \zeta \cdot y} \tilde{u}(\zeta) d^{n} \zeta \\
& =\iint p(y, \zeta) e^{i \zeta \cdot\left(y-y^{\prime}\right)} u\left(y^{\prime}\right) d y^{\prime} d \zeta
\end{aligned}
$$

where $u(y) \in \mathrm{C}_{0}^{\infty}(Y)$ and $d \zeta=d \zeta_{1} d \zeta_{2} \ldots, d \zeta_{n}, d y=d y_{1} d y_{2} \ldots, d y_{n}$. The adjoint of this operator, denoted by $P^{\dagger}$, can be defined as

$$
P^{\dagger} u(y)=(2 \pi)^{-n} \iint p^{*}\left(y^{\prime}, \zeta\right) e^{i \zeta \cdot\left(y-y^{\prime}\right)} u\left(y^{\prime}\right) d y^{\prime} d \zeta
$$

This operator belongs to a more general class of pseudo-differential operators defined by

$$
P u(y)=\iint p\left(y, y^{\prime}, \zeta\right) e^{i \zeta \cdot\left(y-y^{\prime}\right)} u\left(y^{\prime}\right) d y^{\prime} d \zeta
$$

where the symbol $p\left(y, y^{\prime}, \zeta\right) \in \mathrm{C}^{\infty}\left(Y \times Y \times \mathbb{R}^{n}\right)$ lies in $\mathrm{S}_{M}^{m}\left(Y \times Y \times \mathbb{R}^{n}\right)$. The adjoint of this operator is defined by the symbol

$$
p^{\dagger}\left(y, y^{\prime}, \zeta\right)=p^{*}\left(y^{\prime}, y, \zeta\right)
$$

Note that the primed variable is to be integrated over. These new operators do not have unique symbols but they do admit of a representation in terms of the former kind of operators as $P=$ $\mathrm{OP}\left(\sigma_{P}(y, \zeta)\right)+R$ where $\sigma_{P}(y, \zeta) \in \mathrm{S}_{M}^{m}\left(Y \times \mathbb{R}^{n}\right)$ and $R$ is an operator with kernel $K_{R}\left(y, y^{\prime}\right) \in \mathrm{C}^{\infty}(Y \times Y)$ such that

$$
\sigma_{P}(y, \zeta)-\sum_{\alpha \in \mathbb{N}^{n},|\alpha|<N-1} \frac{1}{\alpha ! i^{|\alpha|}} \partial_{\zeta}^{\alpha}\left[\partial_{y^{\prime}}^{\alpha} p\left(y, y^{\prime}, \zeta\right)\right]_{y^{\prime}=y} \in \mathrm{S}_{M}^{m-\mu N}\left(Y \times Y \times \mathbb{R}^{n}\right),
$$

where $N>0$ and $\mu$ is smallest element of $M$. The relationship in equation (A5) defines the asymptotic expansion of the symbol and we write

$$
\sigma_{P}(y, \zeta) \sim \sum_{\alpha \in \mathbb{N}^{n}} \frac{1}{\alpha ! i^{|\alpha|}} \partial_{\zeta}^{\alpha}\left[\partial_{y^{\prime}}^{\alpha} p\left(y, y^{\prime}, \zeta\right)\right]_{y^{\prime}=y}
$$


This determines $\sigma_{P}(y, \zeta)$ only up to a smoothing operator. The formula expressing $\sigma_{P^{\dagger}}(y, \zeta)$ in terms of $\sigma_{P}^{*}\left(y^{\prime}, \zeta\right)$ can be obtained by writing

$$
p^{*}\left(y^{\prime}, y, \zeta\right)-\sigma_{P}^{*}\left(y^{\prime}, \zeta\right) \in \mathrm{S}_{M}^{-\infty}\left(Y \times Y \times \mathbb{R}^{n}\right),
$$

and using the asymptotic expansion (A6)

$$
\sigma_{P^{\dagger}}(y, \zeta) \sim \sum_{\alpha \in \mathbb{N}^{n}} \frac{1}{\alpha ! i^{|\alpha|}} \partial_{\zeta}^{\alpha}\left[\partial_{y^{\prime}}^{\alpha} \sigma_{P}^{*}\left(y^{\prime}, \zeta\right)\right]_{y^{\prime}=y},
$$

where $R$ being a smoothing operator does not show up in the asymptotic expansion. The adjoint can be used to write the Fourier transform of $P u(y)$ by noticing that $\left(P^{\dagger}\right)^{\dagger}=P$ so that

$$
P u(y)=(2 \pi)^{-n} \int\left(\int \sigma_{P^{\dagger}}^{*}\left(y^{\prime}, \zeta\right) e^{-i \zeta \cdot y^{\prime}} u\left(y^{\prime}\right) d y^{\prime}\right) e^{i \zeta \cdot y} d \zeta
$$

Consider the inner product defined by $\langle u \mid v\rangle=\int u^{*} v d y$. An expression of the form $\operatorname{Re}\langle u \mid P u\rangle$ arises in establishing the stability of the ABCs. This can be computed by observing

$$
2 \operatorname{Re}\langle u \mid P u\rangle=\langle u \mid P u\rangle+\left\langle u \mid P^{\dagger} u\right\rangle
$$

Define $2 Q=\left(P+P^{\dagger}\right)$ and using Plancheral's theorem, we have

$$
\operatorname{Re}\langle u \mid P u\rangle=(2 \pi)^{-n} \iint d \zeta d y^{\prime} \tilde{u}^{*}(\zeta) \sigma_{Q}\left(y^{\prime}, \zeta\right) u\left(y^{\prime}\right) e^{-i \zeta \cdot y^{\prime}} .
$$

If $\sigma_{Q}(y, \zeta)$ is independent of $y$, then

$$
\operatorname{Re}\langle u \mid P u\rangle=(2 \pi)^{-n} \int d \zeta|\tilde{u}(\zeta)|^{2} \sigma_{Q}(\zeta)
$$

The sign of this expression can solely be decided by the sign of $\sigma_{Q}(\zeta)$. If $\sigma_{Q}(y, \zeta)=\phi\left(y_{1}\right) \sigma(\tilde{\zeta})$ where $\tilde{y}=\left(y_{2}, y_{3}, \ldots, y_{n}\right) \in \mathbb{R}^{n-1}$ and $\tilde{\zeta}=\left(\zeta_{2}, \zeta_{3}, \ldots, \zeta_{n}\right) \in \mathbb{R}^{n-1}$ then

$$
\operatorname{Re}\langle u \mid P u\rangle=(2 \pi)^{-(n-1)} \iint d y_{1} d \tilde{\zeta}\left|\mathscr{F}_{\tilde{y}} u\left(y_{1}, y\right)\right|^{2} \phi\left(y_{1}\right) \sigma(\tilde{\zeta}) .
$$

On account of the ambiguity in the knowledge of the exact symbol, the conclusion remains valid only up to an infinitely smoothing operator (except for the special cases where the symbol is of principle type). Some relevant examples are discussed below.

- Fractional operators with symbol $p(t, \xi)=\eta(i \xi)^{\alpha}$ (where $t \in \mathbb{R}$ with covariable $\xi \in \mathbb{R} \backslash\{0\}$ ):

$$
\begin{aligned}
\sigma_{Q}(\xi) & \sim \frac{1}{2}\left[\eta(i \xi)^{\alpha}+\eta^{*}(-i \xi)^{\alpha}\right] \\
& =|\eta||\xi|^{\alpha} \cos \left[\frac{\pi \alpha}{2} \operatorname{sgn}(\xi)+\arg \eta\right] .
\end{aligned}
$$

- Let $\sigma_{P}=\eta \phi(x)(i \xi)^{\alpha}$ with real valued function $\phi(x)>0, \forall x \in \mathbb{R}$ where the meaning of the variables is same as that of the last example. Then

$$
\begin{aligned}
\sigma_{Q}(x, t, \zeta, \xi) & \sim \frac{1}{2}\left[\eta \phi(x)(i \xi)^{\alpha}+\eta^{*} \phi(x)(-i \xi)^{\alpha}\right] \\
& =|\eta| \phi(x)|\xi|^{\alpha} \cos \left[\frac{\pi \alpha}{2} \operatorname{sgn}(\xi)+\arg \eta\right],
\end{aligned}
$$

so that

$$
\operatorname{Re}\langle u \mid P u\rangle=\frac{|\eta|}{2 \pi} \iint d \xi d x\left|\mathscr{F}_{t}[u(x, t)](x, \xi)\right|^{2} \phi(x)|\xi|^{\alpha} \times \cos \left[\frac{\pi \alpha}{2} \operatorname{sgn}(\xi)+\arg \eta\right] .
$$


- Operators with symbol $p(x, t, \zeta, \xi)=\eta\left(i \xi+i \zeta^{2}\right)^{\alpha}$ (where $(x, t) \in \mathbb{R}^{2}$ with covariables $(\zeta, \xi) \in$ $\left.\mathbb{R}^{2} \backslash\left\{(\xi, \zeta) \in \mathbb{R}^{2}: \xi+\zeta^{2}=0\right\}\right):$

$$
\begin{aligned}
\sigma_{Q}(\zeta, \xi) & \sim \frac{1}{2}\left[\eta\left(i \xi+i \zeta^{2}\right)^{\alpha}+\eta^{*}(-i \xi-i \zeta)^{\alpha}\right] \\
& =|\eta|\left|\xi+\zeta^{2}\right|^{\alpha} \cos \left[\frac{\pi \alpha}{2} \operatorname{sgn}\left(\xi+\zeta^{2}\right)+\arg \eta\right] .
\end{aligned}
$$

In the first two examples, if $-\pi / 2 \leq(\pi \alpha / 2) \operatorname{sgn}(\xi)+\arg \eta \leq \pi / 2$, it is easy to show that the sign of $\sigma_{Q}$ remains fixed. In the last example, the same is true if $-\pi / 2 \leq(\pi \alpha / 2) \operatorname{sgn}\left(\xi+\zeta^{2}\right)+\arg \eta \leq \pi / 2$. These examples are treated exactly in the main body of the paper without resorting to the properties of the pseudo-differential operators (i.e. the ambiguity resulting from the lack of knowledge of the exact symbol is circumvented).

${ }^{1}$ L. D. Menza, "Absorbing boundary conditions on a hypersurface for the Schrödinger equation in a half-space," Appl. Math. Lett. 9, 55-59 (1996).

${ }^{2}$ L. D. Menza, "Transparent and absorbing boundary conditions for the Schrödinger equation in a bounded domain," Numer. Funct. Anal. Optim. 18, 759-775 (1997).

${ }^{3} \mathrm{X}$. Antoine and C. Besse, "Construction, structure and asymptotic approximations of a microdifferential transparent boundary condition for the linear Schrödinger equation," J. Math. Pures Appl. 80, 701-738 (2001).

${ }^{4}$ A. Schädle, "Non-reflecting boundary conditions for the two-dimensional Schrödinger equation," Wave Motion 35, 181188 (2002).

${ }^{5}$ H. Han and Z. Huang, "Exact artificial boundary conditions for Schrödinger equation in $\mathbb{R}^{2}$," Comm. Math. Sci. 2, 79-94 (2004).

${ }^{6} \mathrm{X}$. Antoine, C. Besse, and V. Mouysset, "Numerical schemes for the simulation of the two-dimensional Schrödinger equation using non-reflecting boundary conditions," Math. Comput. 73, 1779-1799 (2004).

${ }^{7}$ C. Zheng, "A perfectly matched layer approach to the nonlinear Schrödinger wave equations," J. Comput. Phys. 227, 537-556 (2007).

${ }^{8}$ R. M. Feshchenko and A. V. Popov, "Exact transparent boundary condition for the parabolic equation in a rectangular computational domain," J. Opt. Soc. Am. A 28, 373-380 (2011).

${ }^{9} \mathrm{X}$. Antoine, C. Besse, and P. Klein, "Absorbing boundary conditions for the two-dimensional Schrödinger equation with an exterior potential. Part I: Construction and a priori estimates," Math. Models Methods Appl. Sci. 22, 1250026 (2012).

${ }^{10} \mathrm{X}$. Antoine, C. Besse, and P. Klein, "Absorbing boundary conditions for the two-dimensional Schrödinger equation with an exterior potential. Part II: Discretization and numerical results," Numer. Math. 125, 191-223 (2013).

${ }^{11} \mathrm{~S}$. Ji, Y. Yang, G. Pang, and X. Antoine, "Accurate artificial boundary conditions for the semi-discretized linear Schrödinger and heat equations on rectangular domains," Comput. Phys. Commun. 222, 84-93 (2018).

${ }^{12} \mathrm{G}$. Pang, Y. Yang, X. Antoine, and S. Tang, "Stability and convergence analysis of artificial boundary conditions for the Schrödinger equation on a rectangular domain," (2018), preprint.

${ }^{13}$ X. Antoine, A. Arnold, C. Besse, M. Ehrhardt, and A. Schädle, "A review of transparent and artificial boundary conditions techniques for linear and nonlinear Schrödinger equations," Comm. Comput. Phys. 4, 729-796 (2008).

${ }^{14} \mathrm{X}$. Antoine, E. Lorin, and Q. Tang, "A friendly review of absorbing boundary conditions and perfectly matched layers for classical and relativistic quantum waves equations," Mol. Phys. 115, 1861-1879 (2017).

${ }^{15}$ G. A. Baker and P. Graves-Morris, "Padé Approximants," (Addison-Wesley publishing Company, Inc., Massachusetts, 1981) Chap. 2, pp. 43-45.

${ }^{16}$ The TBCs derived by Feshchenko and Popov $^{8}$ happen to be a special case where this operation can be carried without having to consider the function $f(x, t)$ over its entire support. The function $f(x, t)$ here refers to the restriction of the field $u(\mathbf{x}, t), \mathbf{x} \in \mathbb{R}^{2}$, to the segments of the boundary of the rectangular domain.

${ }^{17}$ C. Lubich, "Discretized fractional calculus," SIAM J. Math. Anal. 17, 704-719 (1986); "Convolution quadrature and discretized operational calculus. I," Numer. Math. 52, 129-145 (1988); "Convolution quadrature and discretized operational calculus. II," Numer. Math. 52, 413-425 (1988); "On the multistep time discretization of linear initial-boundary value problems and their boundary integral equations," Numer. Math. 67, 365-389 (1994).

${ }^{18} \mathrm{~A}$ distinction must be made between the Padé approximation based methods which are applied to the operator $\left(\partial_{t}-i \Delta_{x}\right)^{\alpha}$ and those applied to the fractional operator formulation of this operator discussed in this paper.

${ }^{19}$ F. A. Milinazzo, C. A. Zala, and G. H. Brooke, "Rational square-root approximations for parabolic equation algorithms," J. Acoust. Soc. Am. 101, 760-766 (1997).

${ }^{20} \mathrm{~A}$. Bamberger, P. Joly, and J. E. Roberts, "Second-order absorbing boundary conditions for the wave equation: a solution for the corner problem," SIAM J. Numer. Anal. 27, 323-352 (1990).

${ }^{21} \mathrm{O}$. Vacus, "Mathematical analysis of absorbing boundary conditions for the wave equation: the corner problem," Math. Comp. 74, 177-200 (2005).

${ }^{22}$ The corner conditions are also incorporated in this equivalent formulation.

${ }^{23}$ We hope that this approach can also be extended to ABCs obtained for the 1D case using microparametrices ${ }^{25}$ in Refs 26 and 27. Further, there are more general type of operators proposed in Ref. 9 that do not appear to be amenable to exact analysis. These possibilities will be explored in a future publication.

${ }^{24}$ L. Nirenberg, "Pseudodifferential operators and some applications," in Lectures on Linear Partial Differential Equations, CBMS Regional Conference Series in Mathematics No. 17 (Amer. Math. Soc., 1973) pp. 19-58. 
${ }^{25}$ R. Lascar, "Propagation des singularités des solutions d'équations pseudo-différentielles quasi-homogénes," Annales de l'Institut Fourier 27, 79-123 (1977).

${ }^{26} \mathrm{~V}$. Vaibhav, "Artificial boundary conditions for certain evolution pdes with cubic nonlinearity for noncompactly supported initial data," J. Comput. Phys. 230, 3205-3229 (2011).

${ }^{27}$ V. Vaibhav, "Microlocal approach towards construction of nonreflecting boundary conditions," J. Comput. Phys. 272, 588607 (2014). 